msh-mss Mathématiques et sciences humaines

169 | Printemps 2005

Varia

\title{
Invariant relations in a finite domain
}

Invariance de relations sur un domaine fini

\section{Luigi Burigana}

\section{(2) OpenEdition}

\section{Journals}

Electronic version

URL: http://journals.openedition.org/msh/2901

DOI: $10.4000 / \mathrm{msh} .2901$

ISSN: 1950-6821

\section{Publisher}

Centre d'analyse et de mathématique sociales de l'EHESS

Printed version

Date of publication: 1 March 2005

ISSN: 0987-6936

\section{Electronic reference}

Luigi Burigana, «Invariant relations in a finite domain », Mathématiques et sciences humaines [Online], 169 | Printemps 2005, Online since 28 March 2006, connection on 23 July 2020. URL : http:// journals.openedition.org/msh/2901; DOI : https://doi.org/10.4000/msh.2901 
Math. \& Sci. hum. / Mathematical Social Sciences $\left(43^{e}\right.$ année, $\mathrm{n}^{\circ} 169,2005(1)$, p. 43-76)

\title{
INVARIANT RELATIONS IN A FINITE DOMAIN
}

\author{
Luigi BURIGANA ${ }^{1}$
}

\begin{abstract}
SUMMARY - Some results of an abstract inquiry into the concept of invariance are presented. The objects to be judged in their possible invariance are relations of arbitrary degrees in one single domain, when they are represented as sets of strings of elements in that domain. The domain itself is presumed to be of finite size, and the transformations with respect to which invariance is judged are injective functions between parts of it. The study focuses on correspondences between algebraic structures of possible relations and algebraic structures of possible transformations, as they are dually interrelated through the invariance condition.
\end{abstract}

KEYWORDS - Invariance, Relation, Boolean operation, Semigroup, Galois connection

RÉSUMÉ - Invariance de relations sur un domaine fini

Une théorie du concept d'invariance est présentée, qui a pour avantage de couvrir aussi des situations où le domaine est fini. Les objets qui sont évalués en ce qui concerne leur éventuelle invariance sont des relations de degré arbitraire internes à un même domaine, représentées comme ensembles de châ̂nes d'éléments d'un tel domaine. Le domaine lui-même est censé être de taille finie et les transformations par rapport auxquelles l'invariance est jugée sont des fonctions injectives entre les sous-ensembles d'un tel domaine. L'étude se concentre sur les correspondances entre les structures algébriques de relations possibles et les structures algébriques de transformations possibles, qui se trouvent reliées par la condition d'invariance. Galois

MOTS CLÉS - Invariance, Relation, Opération booléenne, Semigroupe, Correspondance de

\section{INTRODUCTION}

The condition of invariance plays important roles in several scientific fields. Limiting our references to a few fields which are part of or interact with psychological science, we mention here statistical methodology, in which invariance with respect to symmetries inherent in a statistical model is a qualifying possible property of statistical indices and rules of statistical decision [Lehmann, 1959, ch. 6; Ferguson, 1967, ch. 4]; measurement theory, which applies invariance as the basic criterion for deciding about the possible meaningfulness of statements and computations involving scalar values and for classifying measurement procedures into a hierarchy of types [Adams

\footnotetext{
${ }^{1}$ Università di Padova, Dipartimento di Psicologia Generale, via Venezia 8, I-35131 Padova Italy, luigi.burigana@unipd.it
} 
et al., 1965; Pfanzagl, 1968, ch. 2; Roberts, 1979, ch. 2; Luce et al., 1990, ch. 22; Chiang, 1997; Narens, 2002]; and the psychology of visual perception, a notable part of which is about perceptual constancies - of size, shape, colour, etc. - these being contents in the perceptual scene which keep themselves invariant under certain variations of the visual conditions [Cassirer, 1944; Mundy, Zisserman, 1992, 1994; Pizlo, 1994; van Gool et al., 1994; Walsh, Kulikowski, 1998; Younes, 2004].

In each of its applications, the concept of invariance implies a comparison between a given "object" and certain transformations of the set (or some of the sets) on which that object is constructed. For example, in statistical theory, an object to be judged in terms of invariance may be a formula defining a statistical quantity, a function used as a point estimator, a test of statistical hypotheses, etc.; in measurement theory, an object to be considered in the invariance framework may be a statement involving scalar values, a relation between such values, a formula for computing a derived measure based on such values, etc.; in perceptual theory, objects to be discussed are selected properties of the units apparent in the perceptual scene or relations between those units. These few references suffice to suggest that objects to be considered for invariance are indeed of many and quite different kinds. We may safely presume that such a variety of types is a major factor in the complexity of a hypothetical general theory of invariance, in its full extent.

The objects of invariance to be discussed in this paper are of one definite type: they are relations - of various degrees - between points in a single domain, i.e., relations internal to one domain. Invariance as referred to set-theoretical objects of this sort has already been the subject of formal investigation, mostly in connection with the meaningfulness problem in measurement theory (in particular, [Pfanzagl, 1968]). As compared with the main body of previous research on the topic, our present contribution distinguishes itself in three respects, which are described in the following paragraphs of this introductory section.

First, we are interested in discussing relations, not as isolated objects, but rather as members of a system which is organized by suitable rules for comparing given relations and transforming or combining them into other relations internal to the same domain. Section 2 is devoted to outlining the sort of algebraic system of relations which is referred to in our analyses. Structural features of that system become especially important in Section 4 in which, after defining the invariance condition, we see how invariance is inherited through the lines determined by rules for comparing and combining relations.

Second, we only consider relations that are defined in a finite domain. This is because our purpose is to explore the formal properties of invariance in finite structures, with possible applications to some problems of visual perception and other areas of psychological science (invariance, as it is analysed in geometry, mathematical statistics and measurement theory, refers to geometrical and/or standard numerical spaces, so that existing theoretical results mainly concern structures that are infinite in their cardinality; see, however, [Fishburn, Roberts, 1989]). Choosing the finite case as the object of our study has an immediate consequence concerning the other term in the invariance dyad, i.e., the concept of a transformation. In the standard theory of invariance, transformations are permutations, i.e., injective and 
surjective functions inside the presumed (infinite) domain. Instead, in our analysis transformations are quasi-permutations, i.e., functions which are injective but not necessarily surjective inside the presumed (finite) domain. The concept of a quasipermutation, the reasons justifying its adoption for our analysis, and some of its most elementary properties are discussed in Section 3.

Third, we are also interested in making explicit the duality between relations and transformations as it is established by the invariance condition, which is a bipolar connection in its very logical nature. Moving from a relation or a set of relations, the set of all transformations under which those relations are invariant may be determined; reciprocally, starting from a transformation or a set of transformations, the set may be determined of all relations that are invariant under those transformations. Sections 5 and 6 examine this pair of symmetric movements, from one to the other side of the invariance bipolar paradigm, and explore some of their formal properties and direct implications for the theory of meaningfulness.

\section{RELATIONS}

We begin our theoretical construction by describing one of the two sides of the invariance paradigm, that side being an organized set - an algebraic system - of possible relations of various degrees which are internal to one and the same domain of a finite cardinality. The system to be defined is set-theoretic and combinatorial in its formal aspect and, in describing it, the general concept of a set becomes to be used repeatedly and with different meanings. So we find it expedient to specify the intended meanings by prefixing the word "set" with suitable letters; e.g., "v-set" for a set of values, "d-set" for a set of dimensions, and so on.

\subsection{SET-THEORETIC REPRESENTATION OF A RELATION}

At the conceptual level, a relation is expressed by some well-formed formula $\alpha$ containing - among other things - one or more distinct symbols $x, \ldots, z$, called variables in the logical sense. Variables denote the roles or (empty) positions involved in the relation; their number is the degree of the relation, and their set $J=\{x, \ldots, z\}$ in this study is called its set of dimensions ( $\mathrm{d}$-set, for short; as we shall see, the term "dimension" is naturally called for by expression "multi-dimensional set", which denotes the set-theoretic representation of the relation). Suppose a set $V$ of entities be specified such that those entities make sense if they are individually located in the places of variables $x, \ldots, z$ within formula $\alpha$; these entities may be numbers, people, colours, cities, etc., depending on the semantic universe to which expression $\alpha$ refers. Set $V$ is called a set of values (v-set, for short); it represents the domain for a possible interpretation of relation $\alpha$, and is presumed to be a finite set in this study. A string based on $J$ and $V$ is any assignment of values in $V$ to dimensions in $J$; in other words, a string $s$ is a function from domain $J$ to codomain $V$ :

$$
s=\{(i, s(i)): i \in J\}=(s(x), \ldots, s(z)),
$$


where $s(i) \in V$ for all $i \in J$. So the set of all possible strings based on $J$ and $V$ is the set of all possible functions from $J$ to $V$; in this study, the complete set of strings is called the multidimensional space (md-space, for short) with dimensions in $J$ and values in $V$, and is denoted by $V[J]$ (its standard set-theoretic notation would be $V^{J}$ ).

In these conditions - in particular, the assumption that formula $\alpha$ makes sense if referred to entities in $V$ - for any string $s \in V[J]$, it is possible to decide whether relation $\alpha$ holds or does not hold between entities $s(x), \ldots, s(z)$, as they are replaced to variables $x, \ldots, z$ inside the formula. So we may consider the set of all strings in $V[J]$ that specify generic formula $\alpha$ into true propositions:

$$
\alpha \mid V=\{s \in V[J]: \alpha(s(x), \ldots, s(z)) \text { is true }\} .
$$

This set is called the interpretation of relation $\alpha$ inside domain $V$. In this way, relation $\alpha$, as referred to set $V$, comes to be represented as a multidimensional set (md-set, for short), i.e., a subset of md-space $V[J]$. In this sense, the set of all md-sets - i.e., power set $P(V[J])$ - amounts to the set of all possible interpreted relations with dimensions in $J$ and values in $V$.

As an example, let us consider the following expression, in which the words have their ordinary arithmetical meanings, so that it refers to a semantic universe of numbers:

$$
\begin{aligned}
& \alpha: \text { "the difference between } w \text { and } x \text { is positive } \\
& \text { and half the difference between } y \text { and } z " .
\end{aligned}
$$

Suppose that set $V=\{1,2,3,4,5\}$ is chosen as the domain for a possible interpretation of relation $\alpha$. We see that the interpretation of $\alpha$ in $V$ is given by the following set of 4-tuples:

$$
\begin{aligned}
\alpha \mid V= & \{(2,1,3,1),(2,1,4,2),(2,1,5,3),(3,2,3,1),(3,2,4,2) \\
& (3,2,5,3),(4,3,3,1),(4,3,4,2),(4,3,5,3),(5,4,3,1) \\
& (5,4,4,2),(5,4,5,3),(3,1,5,1),(4,2,5,1),(5,3,5,1)\} .
\end{aligned}
$$

It is meant that each 4-tuple describes a function from d-set $J=\{w, x, y, z\}$ to $\mathrm{v}$-set $V$, places in the 4-tuple being associated with letters in $J$ as these are ordered in the alphabetic fashion. Thus, for example, 4-tuple $(2,1,3,1)$ specifies function $\{(w, 2),(x, 1),(y, 3),(z, 1)\}$, 4-tuple $(2,1,4,2)$ function $\{(w, 2),(x, 1),(y, 4),(z, 2)\}$, and so on. Interpretation $\alpha \mid V$ is a subset of md-space $V[J]$, which is a set of $5^{4}=625$ strings.

Being a function from set $J$ to set $V$, each string $s$ has a range $R(s)$ which is a subset of $V$, and a kernel $K(s)$ which is a partition of $J$ (we use term "kernel" in accordance with [Aigner, 1979, p. 5]). Any two strings $s, t$ are said to be equivalent to each other (notation: $s \sim t$ ) if they share the same kernel; in other words:

$$
\begin{aligned}
& s \sim t \text { iff } K(s)=K(t) \\
& \text { iff } s(i)=s(j) \text { if and only if } t(i)=t(j) \text {, for all } i, j \in J \text {. }
\end{aligned}
$$


Quotient set $V[J] / \sim$ may be considered, which is the set of all equivalence classes in md-space $V[J]$ according to equivalence $\sim$. There is a one-to-one correspondence between that quotient set and a suitable subset of the set of all partitions of d-set $J$, i.e., the partition lattice of $J$ (when $|V| \geq|J|$ then that correspondence covers the entire partition lattice).

Suppose that $J$ and $L$ are two d-sets of equal cardinality $(|J|=|L|)$, e is a bijective function from $L$ onto $J$, and $s$ is a string based on $J$ and $V$. Symbol se denotes a string based on $L$ and $V$; precisely, se is obtained by composing function $e$ with function $s$, so that:

$$
s e(l)=s(e(l)), \text { for all } l \in L .
$$

In the special case $L=J$, function $e$ acts as a reordering rule: for each $i \in J$, value $s(i)$, which occupies position $i$ in the original string $s$, comes to occupy position $e^{-1}(i)$ in the transformed string se. Further, suppose that an md-set $X$ is given, which is a subset of md-space $V[J]$; symbol $X e$ denotes the set of all transformed strings which may be obtained by composing function $e$ with each of the strings in $X$, which means:

$$
X e=\{s e: s \in X\} .
$$

It is immediately recognized that $X e$ is itself an md-set; it is a subset of md-space $V[L]$. Any two md-sets $X \subseteq V[J]$ and $Y \subseteq V[L]$ are said to be convertible with each other in case $Y=X e$ for some bijective function $e$ from $L$ onto $J$.

Shifting our perspective, let us now suppose that $f$ is a function, the domain and range of which are subsets of value set $V$, and that $s$ is a string in $V[J]$, such that its range is a subset of the domain of $f$. Symbol $f s$ is then used to denote a transformed string in $V[J]$, which is derived by composing function $s$ with function $f$, so that:

$$
f s(i)=f(s(i)), \text { for all } i \in J .
$$

We may say that, in this context, function $f$ acts as a substitution rule: in each position $i$ (for $i \in J$ ), value $s(i)$ is replaced by value $f(s(i)$ ), thus transforming string $s$ into string $f s$. It is directly seen that this sort of transformation, acting on values rather than on dimensions, offers a simple way of characterizing equivalence between strings, as defined in (2): any two strings $s, t$ in $V[J]$ are equivalent to each other if and only if $t=f s$ for some $f$, which is a bijection from range $R(s)$ onto range $R(t)$.

Now let us suppose that $J$ and $L$ are disjoint sets of dimensions. For any two strings $s$ in $V[J]$ and $t$ in $V[L]$, their catenation $s+t$ may be considered, defined as:

$$
(s+t)(i)=s(i) \text { or }=t(i) \text {, depending on whether } i \in J \text { or } i \in L \text {. }
$$

This equation unambiguously defines a function from d-set $J \cup L$ to v-set $V$, since $J$ and $L$ are presumed to be disjoint sets; so catenation $s+t$ is a string in $V[J \cup L]$. It is also seen that catenation $s+t$ may be described as the union between $s$ and $t$, 
when the involved strings are viewed as sets (being functions, they are sets of pairs). In the presumed context, three different md-spaces $V[J], V[L]$ and $V[J \cup L]$ come into play, linked together by the following equation:

$$
V[J \cup L]=\{s+t: s \in V[J], t \in V[L]\}
$$

the resulting space $V[J \cup L]$ is a sort of direct product of spaces $V[J]$ and $V[L]$ on smaller sets of dimensions.

\subsection{BOOLEAN OPERATIONS ON RELATIONS}

Dealing with relations and their invariance we wish to bring to the foreground the generative side of the problem, which concerns the fact that, based on some given relations, some other relations may be defined through suitable rules for transforming or combining the given objects. The rules we consider in this paper come down to the Boolean operators, which are given by the logical connectives of negation, conjunction and disjunction in the linguistic expression of relations, and by the set-theoretic operations of complementation, intersection and union in the representation of those same relations as md-sets. As is pointed out in the conclusions to the paper, besides Boolean operators there are other important rules which may be applied for transforming or combining given relations into new relations in the same domain; in particular, a couple of projection operations, which correspond to the existential and universal quantifiers in a logical predicate calculus. By this we wish to emphasize that the algebra of relations which we will define and afterwards use as a tool in our analyses is a limited one, for the sake of simplicity.

Suppose that $J, L$ are d-sets and $X \subseteq V[J], Y \subseteq V[L]$ are md-sets representing some two relations of suitable degrees inside domain $V$. Complement $X^{c}$ of md-set $X$ is simply defined as the set of all strings in md-space $V[J]$ that are not in $X$, which means that $X^{c}$ is itself a subset of $V[J]$ and is determined by the following equation:

$$
X^{c}=\{s \in V[J]: \operatorname{not}(s \in X)\}
$$

Intersection $X \cap Y$ of the two given md-sets is defined as the set containing every string in $V[J \cup L]$, such that both its part on d-set $J$ is in $X$ and its part on d-set $L$ is in $Y$; in other terms, by applying catenation and using symbols $p, q, r$ to refer to arbitrary strings in $V[J \backslash L], V[J \cap L]$ and $V[L \backslash J]$ respectively, the intersection is specified by the following equation:

$$
X \cap Y=\{p+q+r: p+q \in X \text { and } q+r \in Y\} .
$$

In a dual way, union $X \cup Y$ is defined as the set comprising each string in $V[J \cup L]$, such that either its part on $J$ is in $X$ or its part on $L$ is in $Y$ (or both conditions are satisfied), so that, by using the notation just introduced, the following equation holds true:

$$
X \cup Y=\{p+q+r: p+q \in X \text { or } q+r \in Y\}
$$


Thus defined, both intersection $X \cap Y$ and union $X \cup Y$ are subsets of mdspace $V[J \cup L]$. These are special cases of a general rule: the d-set of a relation which is produced through one or more Boolean operations starting from some given relations is the union of the d-sets at the basis of these relations; this is because any compound relation involves each variable that is involved in at least one of the compounding relations. This comment makes it clear that, in combining some given relations to generate a new relation, consideration must be given not only to the kinds of operations that are applied in the generation process, but also to the sets of dimensions involved in the relations to be combined, and the links of coincidence or non-coincidence between those dimensions. In the linguistic expression of relations, coincidence or non-coincidence of dimensions are directly specified as identity or non-identity of the symbols denoting the variables involved in the relations.

As an example, let us again consider formula (1), which expresses a relation $\alpha$ on four variables (a quaternary relation). In addition, let the following formulas be introduced:

$\beta$ : " $u$ differs from the product of $w$ and $x "$

$\zeta:$ : $v$ differs from the product of $y$ and $z$,

which express two ternary relations which are equivalent in their meanings but different in the sets of variables they involve. Now consider the following composite expression:

$$
\eta: " \alpha \text { and }(\text { not } \beta \text { or not } \zeta) "
$$

which reads:

"the difference between $w$ and $x$ is positive and half the difference between $y$ and $z$ and ( $u$ does not differ from the product of $w$ and $x$, or $v$ does not differ from the product of $y$ and $z^{\prime \prime}$ ).

This is a relation on six variables (a 6 -ary relation) which is obtained by combining $\alpha$, $\beta$ and $\zeta$ through Boolean logical connectives (first negation of $\beta$ and $\zeta$; then disjunction of these negations; and lastly conjunction of $\alpha$ and the obtained disjunction). Now suppose that a certain set $V$ is chosen as the domain for possible interpretations of the stated relations. Then the set-theoretic representations of relations $\alpha$, $\beta, \zeta$ will be, in order, a subset $X$ of $V[\{w, x, y, z\}]$, a subset $Y$ of $V[\{u, w, x\}]$ and a subset $Z$ of $V[\{v, y, z\}]$. In its turn, composite relation $\eta$ will be represented by md-set $X \cap\left(Y^{c} \cup Z^{c}\right)$, which is a subset of md-space $V[\{u, v, w, x, y, z\}]$. Simple computations show that, if $V=\{1,2,3,4,5\}$ (as in the previous example), then the interpretation of $\eta$ is the following set of 506 -tuples:

$$
\begin{aligned}
\eta \mid V= & X \cap\left(Y^{c} \cup Z^{c}\right) \\
= & \{(2, o, 2,1,3,1): o \in V\} \cup\{(2, o, 2,1,4,2): o \in V\} \cup\{(2, o, 2,1,5,3): o \in V\} \cup \\
& \{(o, 3,2,1,3,1): o \in V\} \cup\{(o, 3,3,2,3,1): o \in V\} \cup\{(o, 3,4,3,3,1): o \in V\} \cup \\
& \{(o, 3,5,4,3,1): o \in V\} \cup\{(o, 5,3,1,5,1): o \in V\} \cup\{(o, 5,4,2,5,1): o \in V\} \cup \\
& \{(o, 5,5,3,5,1): o \in V\} .
\end{aligned}
$$


Each of these 6-ples specifies a function from d-set $\{u, v, x, y, w, z\}$ to v-set $\{1,2,3,4,5\}$ (e.g., $(2,1,2,1,3,1)$ specifies $\{(u, 2),(v, 1),(w, 2),(x, 1),(y, 3),(z, 1)\})$.

As a factor of coherence in the algebra of relations defined here, let us introduce the idea of a general set of dimensions (general d-set). Such a set, here denoted by $N$, is conceived as a set of finite cardinality but large enough so that any variable in any relation that may be considered during a certain course of analyses may find a distinct identity in $N$. From now on, when referring to any md-set $X$ as a subset of some md-space $V[J]$, we explicitly or implicitly presume that specific d-set $J$ is a subset of general d-set $N$; this is of help in comparing or combining relations represented as md-sets, since their possibly different sets of dimensions come to be subsets of the same general set. Having introduced the concept of a general d-set $N$, we are naturally led to consider general md-space $V[N]$, which is the set of all functions from $N$ to $V$ (the adopted set of values).

It is important to note, in this respect, that associated with any md-set $X \subseteq$ $V[J]$ (where $J \subseteq N$ ) there is a unique md-set $X^{*} \subseteq V[N]$, which is called the free expansion of $X$ (on the general d-set) and is perfectly equivalent to $X$ in its substantial meaning and algebraic properties. The formal definition of free expansion is given by the following equation:

$$
\begin{aligned}
X^{*} & =\{s+t: s \in X, t \in V[N \backslash J]\} \\
& =X \cap V[N \backslash J] .
\end{aligned}
$$

This means that $X^{*}$ is the set containing each string based on general d-set $N$, such that its part on the specific d-set $J$ is a string in $X$, while its part on the complementary d-set $N \backslash J$ is free (intersection in the formula corresponds to conjunction "while" in this verbal description; by contrast, note that $X \cup V[N \backslash J]=V[N]$, the whole md-space). Since any subset of $V[J]$, for any $J \subseteq N$, has a unique free expansion as a subset of $V[N]$, we may refer to power set $P(V[N])$ as the set of all md-sets apt to represent relations internal in domain $V$ and of degrees not larger that $|N|$. Note, in particular, the following equations which are obviously true for all $X \subseteq V[J], Y \subseteq V[L], J, L \subseteq N$ :

$$
\begin{aligned}
\left(X^{c}\right)^{*} & =\left(X^{*}\right)^{c} \\
(X \cap Y)^{*} & =X^{*} \cap Y^{*} \\
(X \cup Y)^{*} & =X^{*} \cup Y^{*} .
\end{aligned}
$$

They show that Boolean operations on md-sets (based on possibly different d-sets) may be turned into and performed as Boolean operations on subsets of one and the same md-space (while intersection and union on the left side are those specially defined by Eqs. (5) and (6), intersection and union on the right side are standard operations of set theory).

\subsection{FIELD OF IDENTIFIABLE RELATIONS}

Let $\mathcal{X}=\left\{X_{1}, \ldots, X_{k}\right\}$ be a set of symbols (in the sequel each of them will be used as the label of some md-set). The concept of a Boolean polynomial (or simply a 
polynomial, there being in the present context no risk of ambiguity) on the terms in $\mathcal{X}$ is given by the following recursive definition: (i) each term $X_{h}$ (for $h=1, \ldots, k$ ) is a polynomial; (ii) if $p$ and $q$ are polynomials, then $(p)^{c},(p) \cap(q),(p) \cup(q)$ are also polynomials; (iii) any formula is a polynomial only if it can be constructed through a finite sequence of applications of rules (i) and (ii) specified above. Let $\mathcal{B}(\mathcal{X})$ be the class of all Boolean polynomials on $\mathcal{X}$, so defined; class $\mathcal{B}(\mathcal{X})$ is obviously an infinite set, since in the definition no upper bound is set to the length or complexity of the formulas which are called Boolean polynomials.

Inside class $\mathcal{B}(\mathcal{X})$, two limited subclasses deserve special consideration; they are class $\mathcal{C}(\mathcal{X})$ of minimal Boolean polynomials and class $\mathcal{D}(\mathcal{X})$ of disjunctive normal forms. Definitions are as follows. A minimal Boolean polynomial on $\mathcal{X}$ is any formula $X_{1}^{s_{1}} \cap \cdots \cap X_{k}^{s_{k}}$, in which $X_{h}^{s_{h}}=X_{h}$ or $=X_{h}^{c}$ for $h=1, \ldots, k$ (all $k$ terms in $\mathcal{X}$ concur in the construction of a minimal polynomial, each being involved either in the positive form $X_{h}$ or the negative form $X_{h}^{c}$ ). A disjunctive normal form on $\mathcal{X}$ is any formula $p_{1} \cup \cdots \cup p_{l}$, where $p_{1}, \ldots, p_{l}$ are distinct minimal Boolean polynomials on $\mathcal{X}$. For example, if $\mathcal{X}=\left\{X_{1}, X_{2}, X_{3}\right\}$, then the following expressions are two distinct minimal polynomials:

$$
\begin{aligned}
& X_{1} \cap X_{2}^{c} \cap X_{3}, \\
& X_{1}^{c} \cap X_{2}^{c} \cap X_{3}
\end{aligned}
$$

while the following expressions are two different disjunctive normal forms:

$$
\begin{aligned}
& \left(X_{1} \cap X_{2}^{c} \cap X_{3}\right) \cup\left(X_{1}^{c} \cap X_{2}^{c} \cap X_{3}\right), \\
& \left(X_{1} \cap X_{2} \cap X_{3}\right) \cup\left(X_{1}^{c} \cap X_{2} \cap X_{3}^{c}\right) \cup\left(X_{1} \cap X_{2}^{c} \cap X_{3}^{c}\right) .
\end{aligned}
$$

Note that, by comparison with the general definition of Boolean polynomials, these special formulas are written with a reduced number of parentheses, this being made possible by the associative property of intersection and union; in addition, due to the commutative property of these operations, some permutations of terms in the given expressions do not alter their meaning. In view of the given definitions and of these properties, we see that using the $k$ terms in $\mathcal{X}, 2^{k}$ distinct minimal polynomials and $2^{2^{k}}$ different disjunctive normal forms can be constructed (we also count as disjunctive normal forms the empty formula, with $l=0$, and each of the minimal polynomials, with $l=1$ ).

Now suppose that symbols $X_{1}, \ldots, X_{k}$ in alphabet $\mathcal{X}$ are given definite interpretations, i.e., they are used as labels for precisely defined md-sets: symbol $X_{h}$ (for $h=1, \ldots, k)$ identifies a certain subset of $V\left[J_{h}\right]$, for some $J_{h} \subseteq N$. As a consequence, each polynomial $p$ in $\mathcal{B}(\mathcal{X})$ comes to receive a definite interpretation, which is itself a precisely defined md-set in $V[N]$; the interpretation of $p$ is determined by starting from md-sets $X_{1}, \ldots, X_{k}$ and transforming or combining them by complementation, intersection and union, as given by Eqs. (4)-(6), implementing step by step the "algorithm" which is inscribed in polynomial $p$, in its syntactic form. This means, for example, that, if $X_{1}$ and $X_{2}$ are two given md-sets and $p=\left(X_{1} \cap X_{2}^{c}\right) \cup\left(X_{1}^{c} \cap X_{2}\right)$, then the interpretation of $p$ is determined by first deriving $X_{1}^{c}$ and $X_{2}^{c}$ according to (4), then obtaining $X_{1} \cap X_{2}^{c}$ and $X_{1}^{c} \cap X_{2}$ by means of (5), and finally producing 
$\left(X_{1} \cap X_{2}^{c}\right) \cup\left(X_{1}^{c} \cap X_{2}\right)$ through (6). Any md-set in $V[N]$ which is the interpretation of some polynomial in $\mathcal{B}(\mathcal{X})$ is said to be identifiable on the basis of $\mathcal{X}$, and the complete collection of such identifiable md-sets is here denoted by $\phi(\mathcal{X})$, so that:

$$
\phi(\mathcal{X})=\{X: X \text { is identified by some } p \in \mathcal{B}(\mathcal{X})\} .
$$

Class $\phi(\mathcal{X})$ represents the total identification power of the algebraic system resulting from the presumed selection $\mathcal{X}=\left\{X_{1}, \ldots, X_{k}\right\}$ of initial md-sets and the triad $\{c, \cap, \cup\}$ of Boolean operations: the larger class $\phi(\mathcal{X})$ inside the universe of md-sets in $V[N]$, the higher the identification power of the system. Inside class $\phi(\mathcal{X})$ we may consider, in particular, subclass $\pi(\mathcal{X})$ of non-empty md-sets which are identified by minimal polynomials in $\mathcal{C}(\mathcal{X})$, i.e.:

$$
\pi(\mathcal{X})=\{X: X \text { is identified by some } p \in \mathcal{C}(\mathcal{X}), X \neq \emptyset\}
$$

There are many properties which may be found to be true by examining class $\phi(\mathcal{X})$ as a set-theoretic structure. The next paragraph mentions only those properties that will be of use in the following analyses, cited in a cursory way, since they correspond to well-known results of the theory of Boolean algebras (cf. [Sikorski, 1964; Halmos, Givant, 1998]). For a more direct correspondence with the general theory, reference is made not to class $\phi(\mathcal{X})$ of identifiable md-sets, but rather to class $\phi^{*}(\mathcal{X})=\left\{X^{*}: X \in \phi(\mathcal{X})\right\}$ of free expansions of identifiable md-sets, which is formally equivalent to $\phi(\mathcal{X})$.

First note that the association between polynomials in $\mathcal{B}(\mathcal{X})$ and md-sets in $V[N]$ is a function but not an injective one; in fact, if some given subset of $V[N]$ is identifiable, then there are infinitely many polynomials in $\mathcal{B}(\mathcal{X})$ which are singly apt to identify it: these polynomials prove to be semantically equivalent while being syntactically different. In particular, for any identifiable md-set there is a disjunctive normal form apt to identify it - a fact which determines an upper bound to the number of identifiable md-sets, given by the following inequality:

$$
\left|\phi^{*}(\mathcal{X})\right| \leq 2^{2^{k}}
$$

where $k$ is the number of md-sets in $\mathcal{X}$ (the generators of the structure). By its very definition, class $\phi^{*}(\mathcal{X})$ is closed under complementation, intersection and union (and all other derivable set-theoretic operations), so that it qualifies as a field of sets, i.e., an example of a Boolean algebra; due to this in the sequel we will refer to $\phi^{*}(\mathcal{X})$ (or to $\phi(\mathcal{X}))$ as the field of identifiable $m d$-sets. Subclass $\pi^{*}(\mathcal{X})=\left\{X^{*}: X \in \pi(\mathcal{X})\right\}$ of free expansions of non-empty md-sets identified by minimal polynomials is a partition of general md-space $V[N]$ (this may be understood by generalizing the property that for arbitrary sets $A, B \subseteq C$, those of intersections $A \cap B, A \cap B^{c}$, $A^{c} \cap B, A^{c} \cap B^{c}$ that are non-empty constitute a partition of $C$ ). More to the point, subclass $\pi^{*}(\mathcal{X})$ is the class of atoms in field $\phi^{*}(\mathcal{X})$, i.e., the class of md-sets which are minimal in class $\phi^{*}(\mathcal{X}) \backslash\{\emptyset\}$ as it is partially ordered by inclusion. This implies the following straightforward relation between cardinalities:

$$
\left|\phi^{*}(\mathcal{X})\right|=2^{\left|\pi^{*}(\mathcal{X})\right|} .
$$


There is also an upper bound for the number of md-sets in $\pi^{*}(\mathcal{X})$, which is given by the following inequality:

$$
\left|\pi^{*}(\mathcal{X})\right| \leq 2^{k}
$$

It may happen that the inequality materializes as an equality, i.e., $\left|\pi^{*}(\mathcal{X})\right|=2^{k}$, which turns out to be the case when none of the minimal polynomials in $\mathcal{C}(\mathcal{X})$ identifies the empty md-set. In such a circumstance, equality $\left|\phi^{*}(\mathcal{X})\right|=2^{2^{k}}$ is also true, field $\phi^{*}(\mathcal{X})$ is a free Boolean algebra, and set $\mathcal{X}^{*}=\left\{X_{1}^{*}, \ldots, X_{k}^{*}\right\}$ of initial md-sets is a system of free generators of that algebra.

Stepping up to a higher level of generality, let us now suppose that a variety $\mathcal{U}=\left\{U_{1}, \ldots, U_{m}\right\}$ of primitive md-sets of degrees $n_{1}, \ldots, n_{m}$ is available, each mdset $U_{i}$ (for $i=1, \ldots, m$ ) being specified as a subset of an "ad hoc" md-space $V\left[N_{i}\right]$ with $\left|N_{i}\right|=n_{i}$, but no definite relationship being presumed between specific d-set $N_{i}$ and general d-set $N$. This is the case, for example, when the alphabet of a predicate calculus is defined, containing $m$ predicate letters of degrees $n_{1}, \ldots, n_{m}$, and the $i$-th predicate letter (for $i=1, \ldots, m$ ) is given a certain interpretation in a chosen domain $V$, as a set of $n_{i}$-tuples of elements in $V$ [Mendelson, 1964, §2.1]. Each primitive md-set $U_{i}$, as it is given at the start, has no definite location with respect to general d-set $N$. To give it such a location, we have to specify an injective function $l$ from $N_{i}$ to $N$, and consider the transformed md-set $U_{i} l^{-1}$ which is determined according to (3). For the same primitive md-set $U_{i}$ there are $n ! /\left(n-n_{i}\right)$ ! different possible locations of this sort, where $n=|N|$. Each one of these locations identifies an md-set in $V[N]$ which is convertible with the original md-set $U_{i}$, so that all located copies of $U_{i}$ are convertible with one another, in the sense defined in Subsection 2.1.

Based on these notions, we may determine number $k$ as sum $n ! /\left(n-n_{1}\right) !+\cdots+$ $n ! /\left(n-n_{m}\right) !$ and denote by $\lambda(\mathcal{U}, N)$ the set of $k$ md-sets which may be determined by locating the primitive md-sets in all the possible ways inside $N$, i.e.:

$$
\lambda(\mathcal{U}, N)=\bigcup_{i=1}^{m}\left\{U_{i} l^{-1}: l \text { is an injection from } N_{i} \text { to } N\right\} .
$$

By applying the argument in the previous paragraphs, we may then come to consider field $\phi(\lambda(\mathcal{U}, N))$ of all md-sets in $V[N]$ which are identified by Boolean polynomials with terms in $\lambda(\mathcal{U}, N)$. Such a field is itself the set-theoretic expression of the overall identification power of a system: it is the universe of relations in domain $V$ which can be produced by means of Boolean operations and on the basis of $U_{1}, \ldots, U_{m}$ as primitive relations, these being freely located inside set $N$ of available dimensions.

\section{TRANSFORMATIONS}

Having developed the notions we need about relations of various degrees in a common domain, which are the objects of the invariance condition as it is discussed in this paper, we now turn to considering the other side of the invariance paradigm, which is given by transformations acting inside the presumed domain. 


\subsection{QUASI-PERMUTATIONS OF VALUES}

By a quasi-permutation (q-permutation) in v-set $V$ we mean any injective function the domain and range of which are subsets of $V$. In other words, a q-permutation in $V$ is any binary relation $f=\left\{\left(u_{1}, v_{1}\right), \ldots,\left(u_{p}, v_{p}\right)\right\}$ in $V$ (i.e., a subset of $V^{2}$ ), such that the following condition is satisfied:

$$
u_{i}=u_{j} \text { iff } v_{i}=v_{j} \text {, for all } 1 \leq i, j \leq p .
$$

Sets $I(f)=\left\{u_{1}, \ldots, u_{p}\right\}$ and $O(f)=\left\{v_{1}, \ldots, v_{p}\right\}$ are called the input-range and, respectively, the output-range of the q-permutation. It may be that $I(f)=V=$ $O(f)$, which would mean that function $f$ is a bijection from set $V$ onto itself; in this special case, function $f$ is called a full permutation or, more simply, a permutation on $V$, which is its ordinary name in set theory and algebra.

In standard theorizations on invariance, which mostly refer to domains of infinite cardinality, the transformations involved are permutations, in the regular sense just recalled. This implies that the complete sets of transformations referred to in gauging the invariance of given objects are groups of permutations, which correspond to a fundamental concept in the algebraic theory of groups. However, when dealing with the invariance of objects which are defined on a finite domain, the concept of a permutation as an exhaustive one-to-one function over that domain may cause problems, due to the fact that, for some simple objects built in that domain, the only permutation under which they are invariant is the identity function. For objects of this kind and in this context, any analysis concerning invariance would be devoid of interest at the start, as long as the transformations to be considered were bound to be permutations. Consider, for example, a finite domain $V$ and an object as simple as a linear order $R$ on $V$; it is easily seen that the only transformation $f$ in $V$ that is a permutation and leaves $R$ invariant (i.e., $\left(v, v^{\prime}\right) \in R$ iff $\left(f(v), f\left(v^{\prime}\right)\right) \in R$, for all $v, v^{\prime} \in V$ ) is the identity function on $V$ (i.e., $f(v)=v$, for all $v \in V$ ). It may also be seen that this sort of conclusion is met for other simple objects - relations or operations - definable in a finite domain. One way of avoiding such a possible dead end is to replace the concept of a permutation with a weaker concept on the transformational side of the invariance paradigm. More precisely, the concept of a permutation is replaced by a concept which preserves the idea of a one-to-one substitution rule - distinct elements are replaced by other distinct elements - but is not bound to be exhaustive of domain $V$. This is precisely the concept of a quasipermutation, which is defined as an injective but possibly non-surjective function inside domain $V$.

The set of all q-permutations in $V$ is denoted here by $Q(V)$. An elementary combinatorial computation shows that, if $m$ is the number of points in $V$, then the number of q-permutations in $V$ is as follows:

$$
|Q(V)|=\sum_{i=0}^{m}(m ! /(m-i) !)^{2} / i !
$$

Set $Q(V)$ includes the set of all $m$ ! permutations on $V$ (the so-called "symmetric group" on $V)$; in particular, one member of $Q(V)$ is the identity permutation, denoted here by $\epsilon(\epsilon(v)=v$, for all $v \in V)$. Empty set $\emptyset$ is itself a member of $Q(V)$ : it 
is the empty set of pairs of points in $V$ which vacuously satisfies condition (10). For any q-permutation $f$, its inverse $f^{-1}$ may be considered, which is formed by reversing each of the pairs in $f$; clearly, $f^{-1}$ is itself a q-permutation and $I\left(f^{-1}\right)=O(f)$, $O\left(f^{-1}\right)=I(f)$. Moreover, for any two q-permutations $f, g$, their composition $g \circ f$ (as the composition of two binary relations in $V$ ) may be determined, which is so defined:

$$
g \circ f=\left\{(u, w) \in V^{2}:(u, v) \in f \text { and }(v, w) \in g \text { for some } v \in V\right\} .
$$

Obviously, $g \circ f$ is itself a q-permutation and its ranges are given by the following equations:

$$
I(g \circ f)=f^{-1}(O(f) \cap I(g)), \quad O(g \circ f)=g(O(f) \cap I(g)) .
$$

Note that, if $O(f) \cap I(g)=\emptyset$, then $g \circ f$ is the empty set, which is counted as a q-permutation. Structure $\left(Q(V), \circ^{-1}\right)$ may be considered, formed by set $Q(V)$ of all q-permutations in $V$, the binary operation $\circ$ of composition and the unary operation $^{-1}$ of inversion. This structure is a semigroup with identity (identity permutation $\epsilon$ ) and zero (empty q-permutation $\emptyset)$; it is enriched by operation ${ }^{-1}$ which is obviously involutive $\left(\left(f^{-1}\right)^{-1}=f\right.$, for any $\left.f \in Q(V)\right)$ and together with $\circ$ satisfies the following equation:

$$
(g \circ f)^{-1}=f^{-1} \circ g^{-1}
$$

The structure is not a group, however, since for any q-permutation $f$ which is not a permutation there is no q-permutation $g$ such that $g \circ f=\epsilon$.

While no two distinct permutations on the same set $V$ are comparable by set inclusion - neither of them, as sets of pairs, is a subset of the other - it is obviously possible that two distinct q-permutations may be comparable in that manner. So it makes sense to examine the properties of set $Q(V)$, as it is partially ordered by relation $\subseteq$ of set inclusion. First note, in this regard, that the partially ordered set $(Q(V), \subseteq)$ has a minimum (the empty q-permutation) and no maximum but various distinct maximals (the $m$ ! full permutations on $V$ ). Further note that set $Q(V)$ is downward-complete in the order of inclusion, in that any subset of any q-permutation is itself a q-permutation. As an immediate corollary, set $Q(V)$ is also closed under intersection, which means that the intersection of any two q-permutations is itself a q-permutation.

Subsets of $Q(V)$ that share with it the properties of being closed under composition and inversion and downward-complete in the order of inclusion are called here semigroups of q-permutations in $V$. This name is appropriate, since such subsets of $Q(V)$ are indeed semigroups in the ordinary algebraic sense (cf. [Howie, 1976]). Because of its downward completeness, any semigroup $G$ of q-permutations is fully determined when set $\kappa(G)=\max _{\subseteq} G$ of its maximal terms in the order of inclusion is known, called here the crown of semigroup $G$. The following proposition specifies the characteristics which allow us to decide whether any given set of q-permutations is or is not the crown of some semigroup of q-permutations. 
PROPOSITION 1. Any given set $F$ of q-permutations is the crown of some semigroup of q-permutations if and only if it is closed under inversion, for any $f, g$ in $F$ there is some $h$ in $F$ such that $g \circ f \subseteq h$, and the q-permutations in $F$ are mutually incomparable by inclusion.

Proof. Part "only if" in the proposition is straightforward. The only point which deserves comment is the fact that a q-permutation $f$ is maximal in a semigroup if and only if its inverse $f^{-1}$ is itself maximal in the same semigroup, which proves that the crown must be closed under inversion. To prove part "if", suppose $F$ be a set of q-permutations with the stated properties and put $G=\{g \in Q(V): g \subseteq$ $f$ for some $f \in F$ \} (i.e., $G$ is the order ideal generated by $F$ within $Q(V)$ ). By its very definition, $G$ is downward-complete and has $F$ as its set of maximal terms. Moreover, if $g \subseteq f$ for some $f \in F$, then $g^{-1} \subseteq f^{-1}$ where $f^{-1} \in F$, which shows that $G$ is closed under inversion. Lastly, if $k \subseteq f$ and $l \subseteq g$ for some $f, g \in F$, then $l \circ k \subseteq g \circ f$ where $g \circ f \subseteq h$ for some $h \in F$, so that $l \circ k \subseteq h$ as well, and this proves that $G$ is closed under composition.

Note that crowns in $Q(V)$ only containing (full) permutations are precisely the groups of permutations on domain $V$, in the ordinary algebraic meaning of the concept.

There are various possible semigroups of q-permutations in a given domain. The following proposition is about intersection as applied to such sets.

PROPOSITION 2. If $F$ and $G$ are semigroups of q-permutations, then their intersection $F \cap G$ is itself a semigroup of q-permutations, and coincides with the set of all intersections between q-permutations in $F$ and q-permutations in $G$, i.e.:

$$
F \cap G=\{f \cap g: f \in F, g \in G\} .
$$

The crown of $F \cap G$ is given by the follouing equation:

$$
\kappa(F \cap G)=\max _{\subseteq}\{k \cap l: k \in \kappa(F), l \in \kappa(G)\} .
$$

Proof. The proposition in its first part is immediately accounted for by the fact that closure under inversion and closure under composition are both preserved by intersection of sets of q-permutations, as well as downward completeness in the order of inclusion. Equation (11) is obtained by considering that, if $h \in F \cap G$, then obviously $h \in F, h \in G$ and $h=h \cap h$, and that, for all $f \in F$ and $g \in G$, certainly $f \cap g \in F$ and $f \cap g \in G$, due to the downward completeness of $F$ and $G$. Equation (12) is proved true by noting that, for all $f \in F$ and $g \in G$ there are $k \in \kappa(F)$ and $l \in \kappa(G)$, such that $f \subseteq k$ and $g \subseteq l$, so that $f \cap g \subseteq k \cap l$; in addition, $k \cap l \in F \cap G$ for all $k \in \kappa(F)$ and $l \in \kappa(G)$, due to Equation (11) and inclusions $\kappa(F) \subseteq F, \kappa(G) \subseteq G$.

Note that set $\{k \cap l: k \in \kappa(F), l \in \kappa(G)\}$ certainly includes the crown $\kappa(F \cap G)$ but is not necessarily itself a crown, since it may violate the incomparability condition (cf. Proposition 1). 
Let us denote by $\mathcal{Q}(V)$ the set of all semigroups of q-permutations in $V$, so that $\mathcal{Q}(V)$ is a collection of special (regular) subsets of set $Q(V)$ of all q-permutations in $V$. Proposition 2 in its opening part ensures that $\mathcal{Q}(V)$ is closed under intersection, while it is clear that $Q(V)$ is itself a member of $\mathcal{Q}(V)$, and so $\mathcal{Q}(V)$ is a closure system or Moore family (cf. [Birkhoff, 1967, §V.1]). Then, for any $F \subseteq Q(V)$ the following equation uniquely determines a member of $\mathcal{Q}(V)$ :

$$
\sigma(F)=\bigcap\{G \in \mathcal{Q}(V): G \supseteq F\} .
$$

It is called the semigroup of q-permutations in $V$ generated by $F$. In the practical sense, it is the smallest semigroup of q-permutations containing all q-permutations given in $F$. In its generality, Equation (13) defines a mapping $\sigma$ from power set $P(Q(V))$ into itself, which is the closure operator corresponding to closure system $\mathcal{Q}(V)$.

\subsection{QUASI-PERMUTATIONS ACT AS CLASSIFIERS}

Suppose that $f$ is a q-permutation in $V, J$ a set of dimensions in $N$, and $s$ and $t$ strings in $V[J]$. We say that $s$ is related to $t$ through $f$, and write $s \rightarrow_{f} t$, if string $t$ equals transformed string $f s$. In a formula:

$$
\begin{aligned}
s \rightarrow_{f} t & \text { iff } t=f s \\
& \text { iff } t(i)=f(s(i)), \text { for all } i \in J .
\end{aligned}
$$

Writing $s \rightarrow_{f} t$ therefore means that $t$ can be derived from $s$ through a term-by-term replacement of values using $f$ as the substitution rule. Note that if $s \rightarrow_{f} t$, then $R(s) \subseteq I(f)$ and $R(t) \subseteq O(f)$, i.e., the set of values in $s$ must be covered by the input range of $f$ and the set of values in $t$ by the output range of $f$; this is because strings $s$ and $t$ are presumed to be based on the same d-set $J$. Also note that if $s \rightarrow_{f} t$, then $s \sim t$, i.e., $s$ and $t$ are related by the general equivalence given in (2), since q-permutation $f$ is an injective function.

Once $J \subseteq N$ and $f \in Q(V)$ are fixed, reference may be made to the structure $\left(V[J], \rightarrow_{f}\right)$ as a digraph (directed graph) the points of which are all strings in $V[J]$ and the lines are links induced by $f$ according to (14). If $I=I(f)$ and $O=O(f)$ are respectively the input and output ranges of $f$, then all strings in $I[J] \cup O[J]$ happen to be involved in relation $\rightarrow_{f}$, either as points from which arrows in the digraph are exiting, or as points in which arrows are entering; strings in the difference set $V[J] \backslash(I[J] \cup O[J])$ are isolated points in the digraph. Based on $\rightarrow_{f}$, md-space $V[J]$ comes to be partitioned into a set of weakly connected components; they are the equivalence classes modulo the following equivalence, which is generated on $V[J]$ by relation $\rightarrow_{f}$ :

$$
\begin{gathered}
s \sim_{f} t \text { iff either } s=t \text { or } s T_{0} r_{1} T_{1} \ldots T_{m-1} r_{m} T_{m} t \text { for some } r_{1}, \ldots, r_{m} \in V[J] \\
\text { where either } T_{i}=\rightarrow_{f} \text { or } T_{i}=\leftarrow_{f}, \text { for } i=1, \ldots, m .
\end{gathered}
$$


Each string in $I[J] \cup O[J]$ falls into some block of at least size 2 in that partition, whereas all strings in $V[J] \backslash(I[J] \cup O[J])$ are singleton blocks. As $s \sim_{f} t$ implies $s \sim t$, quotient set $V[J] / \sim_{f}$ is a refinement of quotient set $V[J] / \sim$ within the lattice of all partitions of $V[J]$.

Rather than one single q-permutation, we might choose a set $F$ of q-permutations in $V$ and consider the relation $\rightarrow_{F}$ which it induces on md-space $V[J]$, which is so defined for all $s, t \in V[J]$ :

$$
s \rightarrow_{F} t \text { iff } s \rightarrow_{f} t \text { for some } f \in F \text {. }
$$

In other words, $\rightarrow_{F}$ is obtained as the disjunction of all relations $\rightarrow_{f}$, for $f \in F$. In addition, equivalence $\sim_{F}$ generated by relation $\rightarrow_{F}$ on $V[J]$ may be considered, which is defined by a modified version of (15) where $\rightarrow_{F}$ is substituted for $\rightarrow_{f}$.

PROPOSITION 3. Let $F$ be any set of q-permutations in $V$ and $G=\sigma(F)$ the semigroup of q-permutations generated by $F$ (Eq. (13)). Then equivalences $\sim_{F}$ and $\sim_{G}$ on $V[J]$ (for any $d$-set $J \subseteq N$ ) are identical.

Proof. Certainly $\sim_{F} \subseteq \sim_{G}$, simply because $F \subseteq G$. To prove that the reciprocal inclusion also holds true, first consider that, if $g \in G$, then $g \subseteq g_{1} \circ \cdots \circ g_{m}$ where for each $i=1, \ldots, m$ either $g_{i}=f_{i}$ or $g_{i}=f_{i}^{-1}$, and $f_{1}, \ldots, f_{m}$ are q-permutations to be found in $F$; this is due to the assumption that $G$ is the semigroup of q-permutations generated by $F$. Hence, if $s \rightarrow_{g} t$ for some $g \in G$, then $s \sim_{F} t$ as well, so that $\sim_{G} \subseteq \sim_{F}$ at a more general level. Putting together the two symmetrical inclusions the identity $\sim_{F}=\sim_{G}$ is obtained.

Blocks forming quotient set $V[J] / \sim_{F}=V[J] / \sim_{G}$ are the weakly connected components of digraph $\left(V[J], \rightarrow_{F}\right)$. Their collection is called the partition induced by $F$ on $V[J]$ and denoted by $\rho(F)$, so that:

$$
\begin{aligned}
\rho(F) & =V[J] / \sim_{F} \\
& =\left\{X \subseteq V[J]: X \text { is a weakly connected component in } \rightarrow_{F}\right\} .
\end{aligned}
$$

The blocks in question are comparable with so-called "orbits" of a permutation group, as special subsets of the domain of that group. Note, however, that $G$ may be any semigroup of q-permutations and, more generally, $F$ may be any set of q-permutations in $V$. Also note that, while q-permutations are acting inside $V$ - so that "orbits" should be subsets of $V$ - blocks in the given quotient set are subsets of $V[J]$. Lastly, consider that, as $s \sim_{f} t$ implies $s \sim t$ for all $f \in F$ and $s, t \in V[J]$, equivalence $\sim_{F}$ is included in the basic equivalence $\sim$ specified by (2), so that partition $\rho(F)$ is a refinement of quotient set $V[J] / \sim$ within the lattice of all partitions of $V[J]$.

\section{THE INVARIANCE CONDITION}

Having defined both sides of the invariance paradigm - md-sets as representations of relations in a given domain on one hand and q-permutations as transformations in that domain on the other - we are now in a position to introduce the concept of invariance itself, as a relationship between the two. 


\subsection{INVARIANT RELATIONS AND RESPECTFUL TRANSFORMATIONS}

Let $X$ be any md-set $(X \subseteq V[J]$ for some $J \subseteq N)$ and $f$ any q-permutation in $V$; let $I=I(f)$ and $O=O(f)$ be the input and output ranges of $f$. We say that $X$ is invariant under $f$ - and, reciprocally, $f$ is respectful of $X$ - if the following composite condition holds true:

$$
\begin{aligned}
& \text { for all } s \in I[J], \text { if } s \in X \text { then } f s \in X \text { and } \\
& \text { for all } s \in O[J] \text {,if } s \in X \text { then } f^{-1} s \in X
\end{aligned}
$$

Restriction " $s \in I[J]$ " as an opening clause in the first part is inserted to ensure that term $f s$ does exist (that term, i.e., the composition of function $s$ with function $f$ is not defined when the range $R(s)$ of the former is not covered by the input range $I(f)$ of the latter). Restriction " $s \in O[J]$ " in the second part is due to a similar reason. A more compact expression of the same concept is:

$$
X \text { is invariant under } f \text { iff } f(X \cap I[J])=X \cap O[J],
$$

where $f Y$, for any md-set $Y \subseteq I[J]$, stands for the set $\{f s: s \in Y\}$ of transformed strings.

In order to comment on the meaning of this concept, let us presume that $X$ is the set-theoretic representation of a relation $\alpha$ on a set of variables $J=\{x, \ldots, z\}$ as that relation is applied to a certain domain $V$, and that $f$ is a rule for substituting values in $V$ for other values in $V$ in a one-to-one manner. Then the first (resp., the second) part of (18) means that, for any assignment of values $(s(x), \ldots, s(z))$ that are in the input (resp., the output) range of rule $f$, the truth value of proposition $\alpha(s(x), \ldots, s(z))$ is the same as the truth value of proposition $\alpha(f(s(x)), \ldots, f(s(z)))$ (resp., of proposition $\alpha\left(f^{-1}(s(x)), \ldots, f^{-1}(s(z))\right)$ ); this means that transformation $f$ acts on aspects which are not substantial for relation $\alpha$, witness the fact that truth values are not affected by that transformation. Note that if $I(f)=V=O(f)-$ i.e., $f$ is a full permutation on $V$ - then equality on the right side of (19) simplifies into $f X=X$, which qualifies $f$ as an automorphism of relation $X$; this is the basic notion in standard theory of invariance, as it is applied to relations in a given domain (cf. [Pfanzagl, 1968, ch.2; Narens, 1981; Luce et al., 1990, ch.22]). The more general property which is expressed by (18) or equivalently by (19) is quite close to the concept of a partial endomorphism of a relation (cf. [Pfanzagl, 1968, ch.1]).

The next statement reveals one important property of invariance, as applied in the framework of md-spaces.

PROPOSITION 4. Let $X$ be any md-set (a subset of $V[J]$, for some $J \subseteq N$ ) and $f$ any q-permutation in $V$. Then $X$ is invariant under $f$ if and only if its free expansion $X^{*}=X \cap V[N \backslash J]$ is itself invariant under $f$.

Proof. First note that $s \in X$ iff $s+t \in X^{*}, f(s+t)=f s+f t$ and $f^{-1}(s+t)=$ $f^{-1} s+f^{-1} t$ for all $s \in V[J]$ and $t \in V[N \backslash J]$. Suppose that $X$ is invariant under $f$, so that condition (18) is true. If $s+t \in X^{*} \cap I[N]$, then $s \in X \cap I[J]$, so that $f s \in X$ due to the first part of (18), whence $f(s+t)=f s+f t \in X^{*}$; similarly, if 
$s+t \in X^{*} \cap O[N]$, then $s \in X \cap O[J]$, which implies $f^{-1} s \in X$ due to the second part of (18), so that $f^{-1}(s+t)=f^{-1} s+f^{-1} t \in X^{*}$; thus composite condition (18) holds true when $X^{*}$ is substituted for $X$, which means that $X^{*}$ is invariant under $f$. A similar argument may be made to verify the reciprocal implication, from the invariance of $X^{*}$ to the invariance of $X$.

Because of the property just proven, in dealing with invariance of given md-sets based on arbitrary and possibly different d-sets in $N$, there is no substantial change if we instead refer to the free expansions of those sets, which are all subsets of the same general md-space $V[N]$. For ease of analysis, we apply this in the following discussion.

One first application of this suggestion lies in expressing and deriving the following characterization of the invariance condition in its basic form.

PROPOSITION 5. Any md-set $X \subseteq V[N]$ is invariant under a q-permutation $f$ if and only if each block in quotient set $V[N] / \sim_{f}$ is either included in or excluded from $X$, which means that the quotient set is a lower bound of bipartition $\left\{X, X^{c}\right\}$ in the lattice of partitions of $V[N]$.

Proof. Suppose that $X$ is invariant under $f$, consider any block $Y$ in $V[N] / \sim_{f}$ such that $|Y|>1$ (if $Y$ is a singleton, the conclusion is trivial) and presume that there is $s \in Y \cap X$. If $t$ is any other string in $Y$, then $s \sim_{f} t$, so that due to (14) and (15), there are strings $r_{0}, r_{1}, \ldots, r_{m}$ such that $r_{0}=s, r_{m}=t$ and either $r_{i}=f r_{i-1}$ or $r_{i-1}=f r_{i}$ for all $i=1, \ldots, m$. On account of formula (18), which defines invariance, starting from hypothesis $s \in X$ and through an inductive procedure we derive that all strings $r_{1}, \ldots, r_{m}$ are in $X$, so that, in particular, $t \in X$. This argument shows that if $X$ is invariant under $f, Y \in V[N] / \sim_{f}$ and $Y \cap X \neq \emptyset$, then $Y \subseteq X$, which is the "only if" part of the theorem. The "if" part is proven true in a similar way.

The concept of invariance can consistently be generalized from comparing one single md-set with one single q-permutation to comparing any given set $\mathcal{X} \subseteq P(V[N])$ of md-sets with any given set $F \subseteq Q(V)$ of q-permutations. The generalization is strictly conjunctive in its logical form and is expressed by saying that $\mathcal{X}$ is invariant under $F$ - and, conversely, $F$ is respectful of $\mathcal{X}$ - if and only if each md-set $X \in \mathcal{X}$ is invariant under each q-permutation $f \in F$. In this way, the idea of invariance, first introduced as a relationship between sets $P(V[N])$ and $Q(V)$, comes to be upgraded as a relationship between sets $P(P(V[N]))$ and $P(Q(V))$. The notions of invariance of a set $\mathcal{X}$ of md-sets under one single q-permutation $f$, and of invariance of one single md-set $X$ under a set $F$ of q-permutations, are special cases of the very general concept just established. [Note that there are other plausible ways of generalizing the invariance condition from one single md-set $X$ to a set $\mathcal{X}$ of md-sets. For example, if $f$ is a full permutation on $V$, one may qualify $\mathcal{X}$ as invariant under $f$ when $f X \in \mathcal{X}$ for all $X \in \mathcal{X}$, rather than $f X=X$ for all $X \in \mathcal{X}$, the latter being a stricter requirement. This observation has to do with the variety of possible "objects" for the invariance condition, a point we made in the introductory section of the paper.] 
As an appendix in Proposition 5, we pointed out that the invariance relationship between an md-set and a q-permutation may be characterized as a refinement relationship between two partitions of the same md-space. This property still holds true for the generalized version of the invariance relationship.

PROPOSITION 6. Let $\mathcal{X}$ be any set of subsets of $V[N]$ and $\pi(\mathcal{X})$ be the set of non-empty subsets of $V[N]$ that are identified by minimal Boolean polynomials based on $\mathcal{X}$ (Equation (8)). Let $F$ be any set of q-permutations in $V$ and $\rho(F)$ be the quotient set of $V[N]$ modulo the equivalence $\sim_{F}$ induced by $F$ on $V[N]$ (Equation $(17))$. Then $\mathcal{X}$ is invariant under $F$ if and only if $\rho(F)$ is a refinement (i.e., a lower bound) of $\pi(\mathcal{X})$ in the lattice of all partitions of $m d$-space $V[N]$.

Proof. Equivalence $\sim_{F}$ is the disjunction of equivalences $\sim_{f}$ induced by all $f \in F$ (cf. Equation (16)), which implies that quotient set $\rho(F)=V[N] / \sim_{F}$ is the join (or least upper bound) of quotient sets $V[N] / \sim_{f}$, for all $f \in F$, within the lattice of all partitions of $V[N]$. It is also easily seen that $\pi(\mathcal{X})$ is a partition and is the meet (or greatest lower bound) of bipartitions $\left\{X, X^{c}\right\}$ for all $X \in \mathcal{X}$, within the same partition lattice. Now, if $\mathcal{X}$ is invariant under $F$, then, by Proposition 5, each quotient $V[N] / \sim_{f}$ is a lower bound of each bipartition $\left\{X, X^{c}\right\}$, so that $\rho(F)$ as a join is a lower bound of $\pi(\mathcal{X})$ as a meet, and this verifies the "only if" part in the theorem. The "if" part is proven true on the basis of these same notions.

\subsection{PRESERVING INVARIANCE AND RESPECTFULNESS}

Let us return to the basic acceptation of invariance, as a relationship between singly considered md-sets and q-permutations. Here we wish to focus on the fact that mdsets are members of a set-theoretic structure, which is equipped with definite criteria for comparing md-sets and transforming or combining them into other md-sets (cf. Subsection 2.2). The same may be said of q-permutations (cf. Subsection 3.1). A rule of invariance preservation expresses the fact that, if any given md-set bears a certain relationship with some other md-sets which have already been shown to be invariant under a particular q-permutation, then that md-set is itself invariant under that q-permutation. In a dual way we may generally describe the notion of a rule of respectfulness preservation.

In the following, some simple rules of invariance preservation (Proposition 7) and of respectfulness preservation (Proposition 8) are made explicit, since they will be of use in our study.

PROPOSITION 7. Let $X, Y \subseteq V[N]$ be md-sets and $f$ a q-permutation in $V$.

(i) If $X$ is convertible with $Y$ and invariant under $f$, then $Y$ itself is invariant under $f$.

(ii) If $X$ and $Y$ are invariant under $f$, then $X^{c}, X \cap Y$ and $X \cup Y$ are all invariant under $f$.

Proof. (i) The hypotheses that $X$ is convertible with $Y$ and invariant under $f$ mean that $Y=X e$ for some permutation $e$ on d-set $N$ and that $s \in X$ implies $f s \in X$ for all $s \in I[N]$ and $f^{-1} s \in X$ for all $s \in O[N]$, where $I=I(f)$ and $O=O(f)$. 
Then for all $s \in I[N]$, if $s \in Y$, then $s e^{-1} \in X$, so that $f s e^{-1} \in X$, whence $f s \in Y$; similarly, for all $s \in O[N]$, if $s \in Y$, then $f^{-1} s \in Y$; thus both parts of (18) hold true when $Y$ is substituted for $X$. (ii) Suppose that $X$ and $Y$ are both invariant under $f$. For each $s \in I[N]$, if $s \in X^{c}$, i.e. $s \notin X$, then $f s \notin X$ due to the second part of (18), as $f s \in O[N]$ and $s=f^{-1} f s$, so that $f s \in X^{c}$; similarly, for each $s \in O[N]$, if $s \in X^{c}$, then $f^{-1} s \notin X$ due to the first part of (18), so that $f^{-1} s \in X^{c}$; thus both parts of (18) are satisfied when $X^{c}$ is substituted for $X$. Furthermore, for all $s \in I[N]$, if $s \in X \cap Y$, then $f s \in X$ and $f s \in Y$, whence $f s \in X \cap Y$, and similarly for all $s \in O[N]$, if $s \in X \cap Y$, then $f^{-1} s \in X \cap Y$, so that both parts of (18) hold true for $X \cap Y$. A parallel argument shows the invariance of $X \cup Y$.

PROPOSITION 8. Let $f, g$ be q-permutations in $V$ and $X \subseteq V[N]$ a md-set.

(i) If $g \subseteq f$ and $f$ is respectful of $X$, then $g$ too is respectful of $X$.

(ii) If $f$ and $g$ are respectful of $X$, then $f^{-1}$ and $g \circ f$ are also respectful of $X$.

Proof. (i) Note that $g \subseteq f$ implies $I^{\prime}[N] \subseteq I[N]$ and $O^{\prime}[N] \subseteq O[N]$, where $I, I^{\prime}$ and $O, O^{\prime}$ are the input-ranges and the output-ranges of $f$ and $g$, respectively, and $g s=f s$ for all $s \in I^{\prime}[N]$ as well as $g^{-1} s=f^{-1} s$ for all $s \in O^{\prime}[N]$. (ii) The equivalence between the respectfulness of $f$ and of $f^{-1}$ is directly due to the duality in (18). Let $f$ and $g$ be any two q-permutations, refer to their composition $g \circ f$, and denote by $I, I^{\prime}, I^{\prime \prime}$ their input-ranges, by $O, O^{\prime}, O^{\prime \prime}$ their output-ranges, so that $I^{\prime \prime}=f^{-1}\left(O \cap I^{\prime}\right)$ and $O^{\prime \prime}=g\left(O \cap I^{\prime}\right)$. Suppose that both $f$ and $g$ are respectful of some given md-set $X$ and consider any $s \in X \cap I^{\prime \prime}[N]$; then $f s \in X \cap I^{\prime}[N]$ (due to the first part of $(18)$ as referred to $f$ ) and $(g \circ f) s=g(f s) \in X$ (due to the first part of (18) as referred to $g$ ). In a similar way it is proved that $s \in X \cap O^{\prime \prime}[N]$ implies $(g \circ f)^{-1} s=f^{-1}\left(g^{-1} s\right) \in X$. So both parts of (18) hold true when $g \circ f$ is substituted for $f$.

The following proposition is a completion theorem, stating that the invariance relationship between a set of md-sets and a set of q-permutations generally implies the same relationship between larger regular sets, which are extensions of the sets initially given.

PROPOSITION 9. Let $\mathcal{X} \subseteq P(V[N])$ be a set of md-sets and $F \subseteq Q(V)$ a set of $q$ permutations in $V$. Furthermore, let $\phi(\mathcal{X})$ be the field of $m d$-sets that are identifiable on the basis of $\mathcal{X}(E q .(7))$ and $\sigma(F)$ the semigroup of q-permutations generated by $F$ (Eq. (13)). If $\mathcal{X}$ is invariant under $F$, then $\phi(\mathcal{X})$ is invariant under $\sigma(F)$.

Proof. For each q-permutation $f \in F$, the assumption that all md-sets in $\mathcal{X}$ are invariant under it implies that any md-set identifiable through a Boolean polynomial based on $\mathcal{X}$ is itself invariant under $f$, due to the rules of invariance preservation in part (ii) of Proposition 7. Hence, the whole field of md-sets $\phi(\mathcal{X})$ is invariant under each $f \in F$. In turn, this result implies that $\phi(\mathcal{X})$ is invariant under each q-permutation in the semigroup generated by $F$, due to the rules of respectfulness preservation of Proposition 8. So the whole of field $\phi(\mathcal{X})$ turns out to be invariant under the whole of semigroup $\sigma(F)$. 
Resuming the general perspective suggested in the last two paragraphs of Section 2 , let us suppose that a variety $\mathcal{U}=\left\{U_{1}, \ldots, U_{m}\right\}$ of primitive md-sets is available, each primitive md-set $U_{i}$ being based on an "ad hoc" set $N_{i}$ of $n_{i}=\left|N_{i}\right|$ dimensions $\left(U_{i} \subseteq V\left[N_{i}\right]\right.$, for $\left.i=1, \ldots, m\right)$. Further suppose that a set $F \subseteq Q(V)$ of q-permutations in $V$ is given, and that one succeeds in verifying that each primitive md-set $U_{i}$ in $\mathcal{U}$ is invariant under each q-permutation $f$ in $F$, in that it satisfies condition (18) with $N_{i}$ and $U_{i}$ in place of $J$ and $X$ respectively. Each one of the $n ! /\left(n-n_{i}\right)$ ! different locations of $U_{i}$ inside the general d-set $N$ is convertible with $U_{i}$, so that the whole family $\lambda(\mathcal{U}, N)$ of $n ! /\left(n-n_{1}\right) !+\cdots+n ! /\left(n-n_{m}\right)$ ! locations of primitive md-sets is invariant under $F$, due to part (i) of Proposition 7. Then, by applying Proposition 9, the conclusion is reached that the whole field $\phi(\lambda(\mathcal{U}, N))$ of md-sets in $V[N]$ that are identifiable on the basis of $\mathcal{U}$ is invariant under the whole semigroup of q-permutations $\sigma(F)$ generated by $F$. In logical parlance, this means that, if we are successful in verifying that a certain variety of primitive predicates as they are applied to a certain domain turn out to be invariant in their truth values under certain transformations of that domain, then we may safely infer that all composite predicates constructible on the basis of those predicates and by use of Boolean operators are themselves invariant under those transformations, and more generally under all transformations in the semigroup generated by them.

\subsection{AN EXAMPLE}

Before presenting the last part of our study, which is about the duality induced by the condition of invariance, we want to illustrate with an example the condition itself as it is defined by (18). Let us consider a set $X=\left\{x_{1}, \ldots, x_{8}\right\}$ of patterns as they are shown in figure 1. Each of these patterns may be conceived as an assignment of different greys to five selected cells in a $3 \times 3$ grid. When cells in the grid are labelled (in the reading order) by letters $a, b, \ldots, h, i$ and the four shades of grey filling the cells are denoted by numbers 1,2,3,4 (a greater number for a lighter shade), then the patterns are faithfully described by the following sets of pairs:

$$
\begin{aligned}
& x_{1}=\{(b, 2),(d, 3),(e, 1),(f, 3),(h, 2)\}, x_{2}=\{(b, 3),(d, 1),(e, 2),(f, 1),(h, 3)\}, \\
& x_{3}=\{(b, 1),(d, 2),(e, 3),(f, 2),(h, 1)\}, x_{4}=\{(b, 2),(d, 3),(e, 4),(f, 2),(h, 3)\}, \\
& x_{5}=\{(b, 1),(d, 2),(e, 4),(f, 2),(h, 1)\}, x_{6}=\{(b, 2),(d, 4),(e, 1),(f, 4),(h, 2)\}, \\
& x_{7}=\{(b, 4),(d, 1),(e, 2),(f, 1),(h, 4)\}, x_{8}=\{(b, 1),(d, 1),(e, 4),(f, 3),(h, 3)\} .
\end{aligned}
$$

In this form each pattern amounts to a function from d-set $J=\{b, d, e, f, h\} \subseteq$ $\{a, b, \ldots, h, i\}=N$ to v-set $V=\{1,2,3,4\}$, so that set $X$ may be viewed as an md-set (a set of strings, as they were called in Subsection 2.1), which is included in md-space $V[J]=V[\{b, d, e, f, h\}]$.

Suppose we intend to make explicit the symmetries which might exist in the given set of patterns. In quite general terms let a symmetry in $X$ be conceived as any injective substitution rule $f$ in v-set $V$ such that, for each pattern $s$ in $X$, if $f$ can be and is applied to $s$, then it transforms $s$ in a pattern $f s$ which is itself in $X$, and the same holds true of its inverse $f^{-1}$ : this, indeed, is the meaning of condition (18), which qualifies $f$ as respectful of $X$ - and, reciprocally, $X$ as 

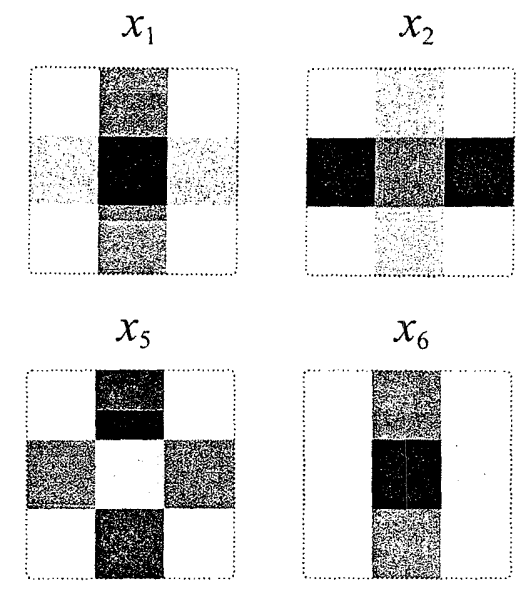
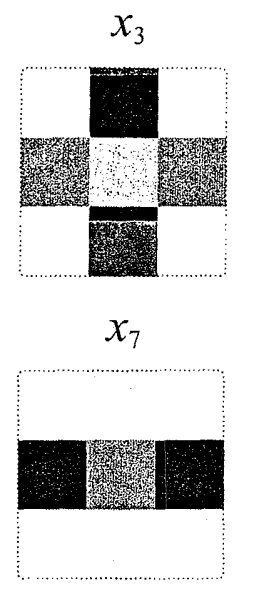
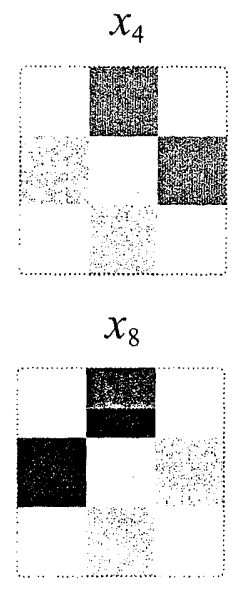

Figure 1.

A set of patterns obtained by colouring with different shades of grey some cells in a square grid.

invariant under $f$. If the search for symmetries is confined to the variety of full permutations on $V$ (i.e., q-permutations of size 4 , as $|V|=4$ ), then the following short result is obtained: the only permutation satisfying (18) for the given $X$ is $\epsilon=\{(1,1),(2,2),(3,3),(4,4)\}$, i.e., the identity permutation on $V$. If instead the search for symmetries may refer more freely to the large variety of q-permutations in $V$, then other and more interesting results are acquired. It is seen, in detail, that both $f_{1}=\{(1,2),(2,3),(3,1)\}$ and $f_{2}=\{(2,1),(3,2),(1,3)\}$ (a pair of qpermutations of size 3 ) are respectful of $X$; more specifically, $f_{1}$ is respectful of part. $\left\{x_{1}, x_{2}, x_{3}\right\}$ in the positive manner (in that $f_{1} x_{1}=x_{2}, f_{1} x_{2}=x_{3}, f_{1} x_{3}=x_{1}$ ) and of part $\left\{x_{4}, x_{5}, x_{6}, x_{7}, x_{8}\right\}$ in the void manner (as neither the input nor the output range of $f_{1}$ covers the range of any of these five patterns, so that equation in (19) as referred to this part comes to be valid in the form $\emptyset=\emptyset)$; the same is true of q-permutation $f_{2}$. Further it is seen that $f_{3}=\{(1,2),(2,4),(4,1)\}$ and $f_{4}=\{(2,1),(4,2),(1,4)\}$ (two other q-permutations of size 3 ) also are respectful of $X$; each of them satisfies condition (18) in the positive manner on part $\left\{x_{5}, x_{6}, x_{7}\right\}$ and in the void manner on part $\left\{x_{1}, x_{2}, x_{3}, x_{4}, x_{8}\right\}$. It is also seen that besides $f_{1}, f_{2}, f_{3}$ and $f_{4}$ there is no other q-permutation of size 3 satisfying condition (18) for md-set $X$, while the 72 q-permutations of size 2, 16 q-permutations of size 1 and the q-permutation of size 0 (the empty set of pairs) comply with that condition, all in the void manner. Thus on the whole there are $1+4+72+16+1=94$ q-permutations being respectful of md-set $X$, and these constitute the semigroup of invariance for $X$, denoted by $\gamma(X)$ (cf. Eq. (20)). The crown $\kappa(\gamma(X))$ of that semigroup is a set of 59 q-permutations, which are $\epsilon, f_{1}, f_{2}, f_{3}, f_{4}$ and all 54 q-permutations of size 2 not included in some of the five larger q-permutations.

To enrich the basis of our example, let us now introduce two more sets of patterns $Y=\left\{y_{1}, \ldots, y_{5}\right\}$ and $Z=\left\{z_{1}, \ldots, z_{5}\right\}$, as they are given in figure 2. By the above criteria these patterns may be described as functions from subsets of the general d-set $N=\{a, b, \ldots, h, i\}$ to $v$-set $V=\{1,2,3,4\}$. Specifically, the following descriptions 
are obtained:

$$
\begin{aligned}
& y_{1}=\{(a, 3),(b, 2),(c, 3),(e, 1)\}, y_{2}=\{(a, 1),(b, 3),(c, 1),(e, 2)\}, \\
& y_{3}=\{(a, 2),(b, 1),(c, 2),(e, 3)\}, y_{4}=\{(a, 2),(b, 4),(c, 2),(e, 1)\}, \\
& y_{5}=\{(a, 2),(b, 1),(c, 2),(e, 4)\}
\end{aligned}
$$

and

$$
\begin{aligned}
& z_{1}=\{(e, 1),(g, 3),(h, 2),(i, 3)\}, z_{2}=\{(e, 2),(g, 1),(h, 3),(i, 1)\}, \\
& z_{3}=\{(e, 3),(g, 2),(h, 1),(i, 2)\}, z_{4}=\{(e, 1),(g, 2),(h, 4),(i, 2)\}, \\
& z_{5}=\{(e, 1),(g, 4),(h, 2),(i, 4)\} .
\end{aligned}
$$

So, set $Y$ amounts to a subset of md-space $V[L]=V[\{a, b, c, e\}]$ and set $Z$ to a subset of md-space $V[M]=V[\{e, g, h, i\}]$.

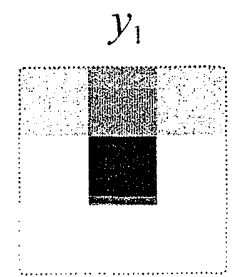

$z_{1}$

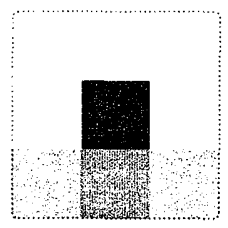

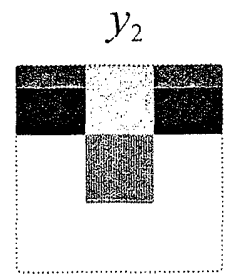

$z_{2}$

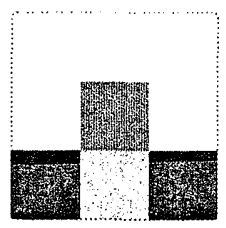

$y_{3}$

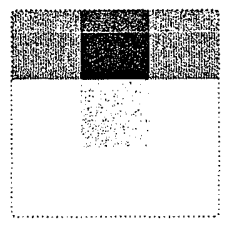

$z_{3}$

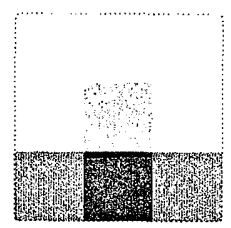

Figure 2.

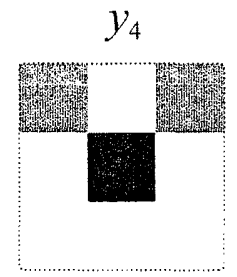

$z_{4}$

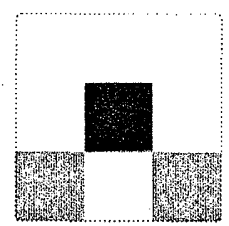

$y_{5}$

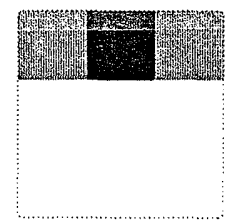

$z_{5}$

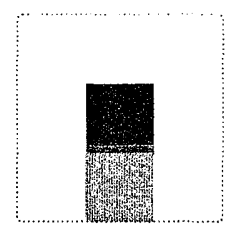

Two more sets of patterns of grey cells in a square grid.

The method used above in determining all q-permutations that are respectful of $X$ may now be applied in dealing with $Y$ and $Z$. It allows us to verify that the invariance semigroup $\gamma(Y)$ for $Y$ is a set of 105 q-permutations, which are $\epsilon, f_{1}$ and $f_{2}$ as defined above, $f_{5}=\{(1,4),(2,2),(4,1)\}$, all 12 q-permutations with inputrange $\{1,3,4\}$ and output-range $\{2,3,4\}$ or vice-versa, and all 89 q-permutations of size less than 3. Crown $\kappa(\gamma(Y))$ is a set of 42 q-permutations, which are $\epsilon$, the 15 q-permutations of size 3 in the semigroup, plus all 26 q-permutations of size 2 not included in any q-permutation of a larger size in the semigroup. Semigroup $\gamma(Z)$ coincides with $\gamma(Y)$ in all respects, except that q-permutation $f_{5}$ is replaced by $f_{6}=\{(1,1),(2,4),(4,2)\}$. Thus, associated with sets of patterns $X, Y$ and $Z$ there are three different semigroups of q-permutations, and we may consider their intersection $\gamma(X) \cap \gamma(Y) \cap \gamma(Z)$, which according to Proposition 2 is itself a semigroup. In view of the above results we see that this last semigroup is a set of 92 q-permutations, which are $\epsilon, f_{1}, f_{2}$ and all q-permutations of size less than 3 . Its crown $\kappa(\gamma(X) \cap \gamma(Y) \cap \gamma(Z))$ is a subset of 63 of those q-permutations. 
Now, sets of patterns $X, Y$ and $Z$ may be taken as the basic terms of an algebraic system, which is developed by applying to them in a recursive way the operations defined by (4)-(6): this perspective of analysis, in which given md-sets are treated not in isolation but rather as members of an organized system, is peculiar to our present study, as pointed out in the introductory section. For example, by applying complementation to $X$ a set $X^{c}$ of $4^{5}-8=1016$ possible patterns is identified, which like $X$ is a subset of md-space $V[J]=V[\{b, d, e, f, h\}]$; by applying union to $X$ and $Y$ a set $X \cup Y$ of $128+320-6=442$ possible patterns is determined, which amounts to a subset of md-space $V[J \cup L]=V[\{a, b, c, d, e, f, h\}]$; by applying intersection to $Y$ and $Z$ the set $Y \cap Z$ of 8 patterns in figure 3 is specified, which is a subset of md-space $V[L \cup M]=V[\{a, b, c, e, g, h, i\}]$. It is easily checked that sets $X, Y$ and $Z$ are independent, in that none of the eight intersections (or minimal Boolean polynomials) $X \cap Y \cap Z, X \cap Y \cap Z^{c}, \ldots, X^{c} \cap Y^{c} \cap Z^{c}$ turns out to be empty. This implies that the system $\phi(X, Y, Z)$ of all md-sets that may be identified through iterated Boolean operations starting from them (cf. Eq. (7)) is a free Boolean algebra with three generators, so that there are $2^{2^{3}}=256$ identifiable sets of patterns.
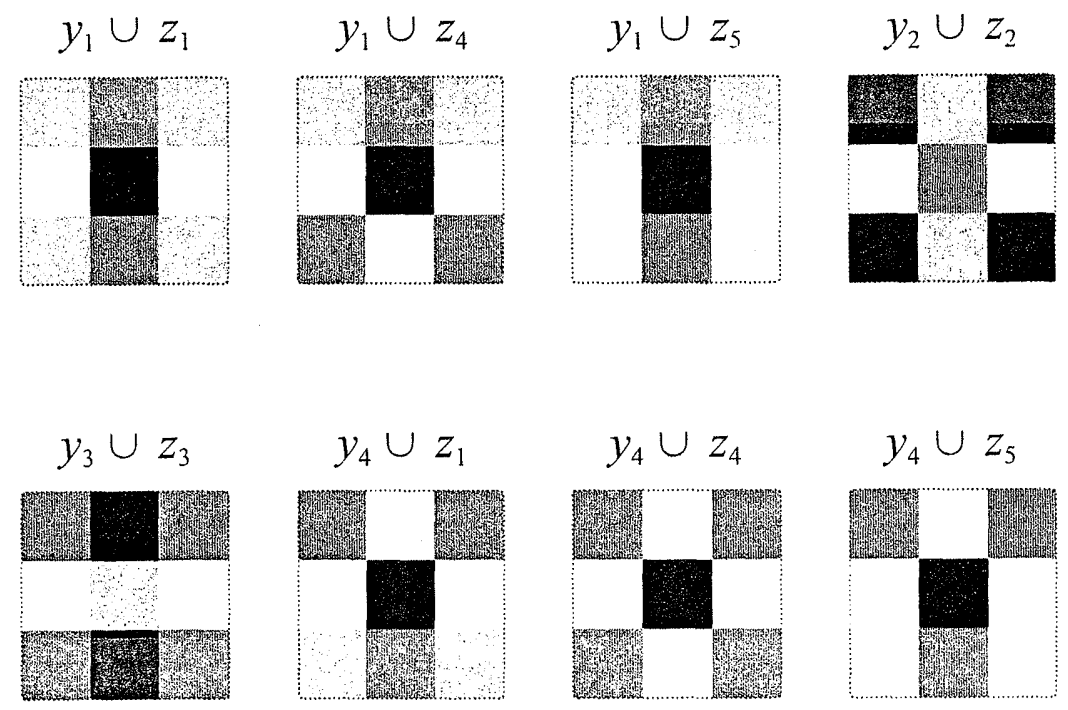

The patterns in the intersection of sets $Y$ and $Z$ as given in figure 2 .

Concerning the algebraic system $\phi(X, Y, Z)$ the following question may be raised: which is the semigroup of q-permutations that are respectful of all 256 md-sets in the system? In other words: which is the complete set of symmetries holding in each of the sets of patterns that may be identified starting from the basic sets $X, Y$ and $Z$ given in the figures? As a corollary of Proposition 7.ii the question receives the following answer: the required semigroup $\gamma(\phi(X, Y, Z))$ is precisely $\gamma(X) \cap \gamma(Y) \cap \gamma(Z)$, i.e., the intersection of the invariance semigroups associated with the three sets of patterns generating the system. 


\section{DUALITY OF RELATIONS AND TRANSFORMATIONS THROUGH INVARIANCE}

In its elementary form, invariance is a relation between two separate universes, which are the universe of md-sets based on given sets of dimensions and values, and the universe of q-permutations inside the set of values. As may be generally done for relations of this sort, through the set-theoretic concept of "conjunctive image" two oppositely directed mappings may be made explicit, one from the power set of the first universe to the power set of the second one, the other from the second to the first power set (cf. [Barbut, Monjardet, 1970; Wille, 1982; Guénoche, 1990]). Defining and analysing this pair of mappings will help in exploring the intrinsic duality of relations and transformations, as it is rooted in the invariance condition.

\subsection{FROM RELATIONS TO RESPECTFUL TRANSFORMATIONS}

Let us refer to any set $\mathcal{X}$ of md-sets in md-space $V[N]$, i.e., any subset of $P(V[N])$. The conjunctive image of $\mathcal{X}$ in the invariance relationship, here denoted by $\gamma(\mathcal{X})$, is the set of all q-permutations in $V$ that are linked with all md-sets in $\mathcal{X}$ in that relationship, i.e.:

$$
\gamma(\mathcal{X})=\{f \in Q(V): f \text { is respectful of each } X \in \mathcal{X}\} .
$$

The following proposition qualifies the general formal aspect of the set thus determined.

PROPOSITION 10. In the stated conditions, $\gamma(\mathcal{X})$ is a semigroup of q-permutations with identity. More precisely, $\gamma(\mathcal{X})$ is the largest semigroup of q-permutations, such that the partition $\rho(\gamma(\mathcal{X})$ ) which it induces on md-space $V[N]$ (Equation (17)) is a refinement of partition $\pi(\mathcal{X})$ induced on the same space by the set of md-sets $\mathcal{X}$ (Equation (8)).

Proof. The first part follows from Proposition 8 and the obvious fact that identity permutation $\epsilon$ on $V$ is respectful of each md-set in $V[N]$. The second part is directly implied by Proposition 6 .

Formally considered, Equation (20) defines a mapping $\gamma$ from $P(P(V[N]))$ (the power set of the set of all md-sets) to $P(Q(V))$ (the power set of the set of all q-permutations). From the conjunctive character of the definition (term "each" in Equation (20)), it is seen that mapping $\gamma$ is antitone with respect to set inclusion, i.e.:

$$
\begin{aligned}
& \text { for all } \mathcal{X}, \mathcal{Y} \subseteq P(V[N]) \\
& \text { if } \mathcal{X} \subseteq \mathcal{Y} \text { then } \gamma(\mathcal{X}) \supseteq \gamma(\mathcal{Y}) .
\end{aligned}
$$

Mapping $\gamma$ is not of injective type. To see this, we consider that if $\mathcal{X}$ and $\mathcal{Y}$ are different families of md-sets that induce the same partition of the md-space $V[N]$ - i.e., $\mathcal{X} \neq \mathcal{Y}$ but $\pi(\mathcal{X})=\pi(\mathcal{Y})$ - then $\gamma(\mathcal{X})=\gamma(\mathcal{Y})$ according to Proposition 10. Note, however, that the non-injectivity of mapping $\gamma$ is of a more radical nature, 
as families $\mathcal{X} \neq \mathcal{Y}$ of md-sets may be found which are partitions of $V[N]$ and such that $\gamma(\mathcal{X})=\gamma(\mathcal{Y})$. Suppose, for example, that $N=\{1,2\}$ and $V=\{a, b, c\}$, and consider the following two partitions of md-space $V[N]=V^{2}$ :

$$
\begin{aligned}
& \mathcal{X}=\{\{a a, a b, b a, b b\},\{a c, b c\},\{c a, c b, c c\}\} \\
& \mathcal{Y}=\{\{a a, a b, b a, b b\},\{a c, b c, c c\},\{c a, c b\}\} .
\end{aligned}
$$

Is easily checked that the q-permutations that are respectful of $\mathcal{X}$ are permutations $\{(a, a),(b, b),(c, c)\}$ and $\{(a, b),(b, a),(c, c)\}$ and all their subsets - as a whole, a semigroup of 14 q-permutations in $V$ - and that these also are the q-permutations being respectful of $\mathcal{Y}$, so that $\gamma(\mathcal{X})=\gamma(\mathcal{Y})$.

According to Proposition 10, the range of mapping $\gamma$ is included in the class of all semigroups of q-permutations in $V$ with identity. Note, however, that there are semigroups in that class which cannot correspond to any family of md-sets through (20), which means that range $\gamma(P(P(V[N])))$ is a proper subset of that class. For example, if $G$ is a semigroup of q-permutations in $V$, such that its crown $\kappa(G)$ is a $k$-transitive permutation group with $k \geq|N|$ (cf. [Dixon, Mortimer, 1996, §2.1|) and is properly included in the symmetric group on $V$, then $G$ cannot be in the range of mapping $\gamma$. This is because, if $\mathcal{X}$ is a family of md-sets which is invariant under all q-permutations in $G$, then $\mathcal{X}$ is also invariant under any permutation on $V$, so that $\gamma(\mathcal{X})=Q(V)$, i.e., the universal semigroup of q-permutations in $V$, which by assumption is different from $G$. The characterization of range $\gamma(P(P(V[N])))$ as a special class of semigroups of q-permutations is a problem in need of scrutiny.

The transition guided by (20) from any given set of md-sets to the complete set of q-permutations that are respectful of them is of a kind of procedure commonly used in mathematics for determining a complete set of transformations in general and a group of permutations in particular, in a selective rather than constructive way. For example, if $X$ is any relation in a domain $V$ and all permutations on $V$ are collected that are respectful of $X$-i.e., permutations $f$ such that $f X=X$ - then a permutation group on $V$ is selectively determined, which is the group of automorphisms of relation $X$. More generally, if a variety $\mathcal{X}=\left\{X_{1}, \ldots, X_{k}\right\}$ of relations in domain $V$ is defined and all permutations on $V$ are considered that prove to be respectful of all those relations, then again a permutation group on $V$ is selectively determined, which is the automorphism group of the relational structure $(V, \mathcal{X})=\left(V, X_{1}, \ldots, X_{k}\right)$ (cf. [Biggs, White, 1979; Kaye, Macpherson, 1994]). Transitions of this sort are key moments in some applications of the invariance theory. For example, in measurement theory, once a numerical relational system is specified as a structure resulting from a numerical domain and one or more relations defined in it, the automorphism group of that system may be determined as the set of all permutations on the domain that are respectful of all relations in the system. The automorphism group then comes to play a critical role in deciding about the meaningfulness of comparisons and computations on the values in the numerical system, depending on whether they comply with suitable invariance conditions under transformations in that group (cf. [Pfanzagl, 1968; Narens, 1981, 1985, 2002; Luce et al., 1990|). 


\subsection{FROM TRANSFORMATIONS TO INVARIANT RELATIONS}

Shifting our perspective, let us now presume that a set $F \subseteq Q(V)$ of q-permutations is given. The conjunctive image of $F$ in the invariance relationship, which we denote by $\delta(F)$, is the set of all md-sets in $V[N]$ that are invariant under all q-permutations in $F$, so that:

$$
\delta(F)=\{X \subseteq V[N]: X \text { is invariant under each } f \in F\} .
$$

In the following proposition, the most immediate formal properties of $\delta(F)$ are made explicit.

PROPOSITION 11. In the presumed conditions, $\delta(F)$ is a field of subsets of space $V[N]$ and is complete with respect to convertibility, i.e., any md-set that is convertible with some md-set in $\delta(F)$ is itself in $\delta(F)$. More precisely, $\delta(F)$ is the set of all unions between blocks in partition $\rho(F)$ induced by $F$ on space $V[N]$, i.e., $\delta(F)$ is the field of sets $\phi(\rho(F))$ (Equations (7) and (17)).

Proof. The first part of this theorem is a direct consequence of Proposition 7. To verify the second part we apply Proposition 5, taking into consideration that $\phi(\rho(F))$ is the largest set of subsets of $V[N]$ such that, for all $X \in \rho(F)$ and $Y \in \phi(\rho(F))$, either $X \subseteq Y$ or $X \cap Y=\emptyset$.

In a way which is specular to that used in the previous section, we may first remark that Equation (22) defines a mapping $\delta$ from $P(Q(V))$ (the power set of the set of all q-permutations in $V)$ to $P(P(V[N]))$. (the power set of the set of all md-sets in $V[N])$. Furthermore, due to the conjunctive logical form of the condition defining $\delta(F)$, that mapping is antitone with respect to set inclusion, i.e.:

$$
\begin{aligned}
& \text { for all } F, G \subseteq Q(V) \\
& \text { if } F \subseteq G \text { then } \delta(F) \supseteq \delta(G) \text {. }
\end{aligned}
$$

The mapping is not injective, as may be inferred from the fact that, if $F$ and $G$ are sets of q-permutations generating the same semigroup of q-permutations, i.e., if $\sigma(F)=\sigma(G)$, then $\delta(F)=\delta(G)$ as well, due to Proposition 9. Proposition 11 makes sure that the range of mapping $\delta$ is inside the class of all fields of sets in $V[N]$. It is properly inside - i.e., not all fields of sets in $V[N]$ are elements of range $\delta(P(Q(V)))$ - and this may be shown in various ways. We may note, for one case, that any field of sets belonging to range $\delta(P(Q(V)))$ must be complete with respect to convertibility (Proposition 11), which is not a property shared by all fields of sets in $V[N]$. We may also consider, for another case, that fields of sets generated by partitions of $V[N]$ like those described in the previous subsection (second paragraph), cannot belong to range $\delta(P(Q(V)))$. Like the characterization of range $\gamma(P(P(V[N])))$, so too the characterization of $\delta(P(Q(V)))$ as a special class of fields of sets in space $V[N]$ is a problem in need of detailed exploration.

The move from a given set of transformations to a complete set of invariant relations, as expressed by (22), is another typical procedure in the theory of invariance and its applications. In measurement theory, for example, once a certain set 
of admissible transformations on the domain of the numerical system is determined - usually the set of all automorphisms of the system - in principle we may refer to the set of all relations in that domain that are meaningful by invariance, in that their truth values are not affected by any of those transformations. Such an ideal complete class of meaningful invariant relations corresponds to the class specified by Equation (22). A similar case may be made concerning statistical methodology. Here, a certain set of legitimate transformations on the sample space may be determined, usually on the basis of symmetries inherent in the presumed statistical model, which may be conceived as a class of possible probability distributions on the sample space (cf. [Bickel, Doksum, 1977]). With reference to that set of legitimate transformations, we may then abstractly consider the set of all statistical objects of a definite kind - statistical relations, indices, decision rules, etc. - that exhibit a suitable sort of invariance under all those transformations. Again, such an exhaustive set of invariant statistical objects corresponds to the set formally defined in (22).

\subsection{A GALOIS CONNECTION}

Sets of md-sets may be compared with one another by set inclusion, which is a partial order, and the same may be done with sets of q-permutations. Thus structures $(P(P(V[N])), \subseteq)$ and $(P(Q(V)), \subseteq)$, which are distinct posets (partially ordered sets), may be considered. Term $\gamma$ as defined by (20) is a mapping from the domain of the first structure to that of the second one; term $\delta$ as specified by (22) is a mapping acting in the opposite direction. Furthermore, mappings $\gamma$ and $\delta$ meet the following conditions, when referred to arbitrary $\mathcal{X}, \mathcal{Y} \in P(P(V[N]))$ and $F, G \in P(Q(V))$ :

$$
\begin{aligned}
& \text { if } \mathcal{X} \subseteq \mathcal{Y} \text { then } \gamma(\mathcal{X}) \supseteq \gamma(\mathcal{Y}) \\
& \text { if } F \subseteq G \text { then } \delta(F) \supseteq \delta(G) \\
& \mathcal{X} \subseteq \delta(\gamma(\mathcal{X})) \\
& F \subseteq \gamma(\delta(F)) .
\end{aligned}
$$

The first two conditions are the antitone properties of $\gamma$ and $\delta$, as previously mentioned and explained (Equations (21) and (23)). The last two conditions are easily verified by noting that, if $X \in \mathcal{X}$, then $X$ is invariant under each $f \in \gamma(\mathcal{X})$ by the very definition of $\gamma(\mathcal{X})$, and similarly, if $f \in F$, then $f$ is respectful of each $X \in \delta(F)$. Because of these four properties, pair $(\gamma, \delta)$ qualifies as a Galois connection between posets $(P(P(V[N])), \subseteq)$ and $(P(Q(V)), \subseteq)$ (cf. [Birkhoff, 1967, §V.8]). The emergence of this standard formal scheme is a new important sign of the duality between md-sets and q-permutations which is established by the invariance condition.

As for any Galois connection, composite mappings $\theta=\delta \circ \gamma$ and $\tau=\gamma \circ \delta$ may be considered, which turn out to be closure operators, the former acting inside $P(P(V[N]))$ and the latter inside $P(Q(V))$. As is true of any closure operator, when partially ordered by set inclusion ranges $\theta(P(P(V[N])))$ and $\tau(P(Q(V)))$ qualify as lattices, inside which the meet operation is simply set intersection; the former is the 
lattice of subsets of $P(V[N])$ that are $\theta$-closed and the latter the lattice of subsets of $Q(V)$ that are $\tau$-closed. Consider the following equations:

$$
\begin{aligned}
& \theta(P(P(V[N])))=\delta(P(Q(V))) \\
& \tau(P(Q(V)))=\gamma(P(P(V[N])))
\end{aligned}
$$

The former is proven by noting that $\theta(\delta(F))=\delta(F)$ for any $F \subseteq Q(V)$, since $\theta(\delta(F)) \supseteq \delta(F)$ due to the extensivity of $\theta$ and $\theta(\delta(F))=\delta(\tau(F)) \subseteq \delta(F)$ due to the extensivity of $\tau$ and to (23); the latter is proven by a similar argument. Thus, we see that the $\theta$-closed subsets of $P(V[N])$ are precisely the fields of subsets of $V[N]$ associated through (22) to arbitrary sets of q-permutations in $V$; symmetrically, the $\tau$-closed subsets of $Q(V)$ are precisely the semigroups of q-permutations in $V$ associated through (20) to arbitrary sets of subsets of md-space $V[N]$.

Operators $\theta$ and $\tau$ are representative of general procedures that may take place in the theory of invariance and its applications. Specifically, when we start from a freely selected variety $\mathcal{X}$ of relations in a domain $V$ and then enlarge it to become the class of all relations in $V$ that are invariant under exactly those transformations in $V$ that are respectful of $\mathcal{X}$, then we are moving from the presumed set $\mathcal{X}$ of basic relations to its $\theta$-closure $\theta(\mathcal{X})$. Similarly, when we first freely choose some set $F$ of transformations in $V$ and then enlarge it to become the set of all transformations in $V$ that are respectful of precisely those relations in $V$ that are invariant under $F$, then we are moving from the initial set $F$ of founding transformations to its $\tau$-closure $\tau(F)$.

\section{INVARIANT VS DEFINABLE RELATIONS}

It is quite easy to verify that mapping $\phi$ as defined by (7) is extensive, monotone and idempotent, so that it is a closure operator in poset $(P(P(V[N])), \subseteq)$. Similarly, mapping $\sigma$ as defined by (13) turns out to be a closure operator in poset $(P(Q(V)), \subseteq)$. Range $\phi(P(P(V[N])))$ of operator $\phi$ is the set of all fields of sets in space $V[N]$, and range $\sigma(P(Q(V)))$ of operator $\sigma$ is the set of all semigroups of q-permutations in $V$ (it is closure system $\mathcal{Q}(V)$ dealt with after proving Proposition 2).

Some comparisons may be made between these closure operators and the closure operators on their same domains that have been discussed in the previous paragraphs. We may consider, for example, the following two relations, which hold true for all $\mathcal{X} \subseteq P(V[N])$ and $F \subseteq Q(V)$, and qualify operator $\phi$ as dominated by $\theta$ and operator $\sigma$ as dominated by $\tau$ :

$$
\begin{aligned}
& \phi(\mathcal{X}) \subseteq \theta(\mathcal{X}) \\
& \sigma(F) \subseteq \tau(F)
\end{aligned}
$$

The former relation is directly implied by the fact that $\phi(\mathcal{X})$ is the smallest field of subsets of $V[N]$ including $\mathcal{X}$ and that $\theta(\mathcal{X})$ is one of such fields of subsets. The latter relation is accounted for by a similar reason. We may also consider the following 
proper inclusions between the ranges of the closure operators:

$$
\begin{aligned}
& \theta(P(P(V[N]))) \varsubsetneqq \phi(P(P(V[N]))) \\
& \tau(P(Q(V))) \varsubsetneqq \sigma(P(Q(V))) .
\end{aligned}
$$

Generic inclusion of the ranges on the left side in the corresponding ranges on the right side is due to the fact that $\theta(\mathcal{X})=\delta(\gamma(\mathcal{X}))$ is a field of subsets of $V[N]$ for each $\mathcal{X} \subseteq P(V[N])$ and $\tau(F)=\gamma(\delta(F))$ is a semigroup of q-permutations in $V$ for each $F \subseteq Q(V)$ (Propositions 10 and 11). Inclusion holds in its strict form, in both cases, because of the facts commented on concerning the ranges of $\gamma$ and $\delta$ in Subsections 5.1 and 5.2.

Of special interest for our purposes is comparison between closure operators $\phi$ and $\theta$, as it may help in clarifying the relationship between two alternative ways of specifying what may be meant by a "meaningful" relation. These ways are known as the "invariance approach" and the "definability approach" to the general problem of meaningfulness (cf. [Pfanzagl, 1968, §2.1; Narens, 1988, 2002; Iverson, Luce, 1998]), the former being more "semantic", the latter more "syntactic" in conception. As applied to the case of relations, both approaches presume that a certain variety $\mathcal{U}=\left\{U_{1}, \ldots, U_{m}\right\}$ of primitive relations have been specified at the start; they may be given as sets of strings of elements in a certain domain $V$, or as primitive predicates on suitable sets of variables to be interpreted in that domain.

The invariance approach was discussed in the previous sections. On the basis of variety $\mathcal{U}$ of primitive relations in the chosen domain $V$, first it determines the set of all transformations in $V$ that are respectful of all relations in $\mathcal{U}$, and then it qualifies as being meaningful any relation in $V$ that proves to be invariant under all those transformations. In formal terms, this means that the class of relations judged to be meaningful in the invariance approach is set $\theta(\lambda(\mathcal{U}, N))$, where $\lambda(\mathcal{U}, N)$ is the set of all locations of relations in $\mathcal{U}$ inside the presumed set $N$ of possible dimensions (Equation (9)).

The definability approach besides a definite set of primitive relations also presumes a certain variety of rules to be accepted as legitimate for generating new relations based on the primitive ones. Then it qualifies to be meaningful not only each of the primitive relations, but also any other relation that may be defined by transforming or combining them through the rules accepted as legitimate for this purpose (cf. [Weitzenhoffer, 1951; Manders, 1981; Narens, 1988; Dzhafarov, 1995]). It is clear that, in this way, the class of meaningful relations comes to depend not only on the selection of the primitive relations but also on the choice of legitimate rules for generating new relations from previous ones. If we suppose that such rules correspond to Boolean operators - negation conjunction and disjunction in the linguistic expression of relations, complementation intersection and union in their set-theoretic representation - then we conclude that the class of relations judged to be meaningful in the definability approach is set $\phi(\lambda(\mathcal{U}, N))$, i.e., the field of subsets of $V[N]$ which is generated by $\lambda(\mathcal{U}, N)$, as defined by $(9)$.

Property (24), which is generally valid, directly implies $\phi(\lambda(\mathcal{U}, N)) \subseteq \theta(\lambda(\mathcal{U}, N))$. This means that any relation which may be proven meaningful in the definability sense - given that the accepted rules of production correspond to Boolean operators 
- also can be proven meaningful in the invariance sense; this is a circumstance already noted in studies on the meaningfulness problem (cf. [Luce, 1978, §4; Luce et al., 1990, §9.3; Dzhafarov, 1995, p. 129]). Furthermore, result (25) suggests that there may be cases in which $\phi(\lambda(\mathcal{U}, N))$ is not in the range of $\theta$, so that $\phi(\lambda(\mathcal{U}, N)) \varsubsetneqq \theta(\lambda(\mathcal{U}, N))$. In a case of this sort, the invariance criterion proves to be more permissive or inclusive than the definability criterion: there are relations that are meaningless in the definability sense but meaningful in the invariance sense.

\section{CONCLUDING COMMENTS}

We have examined some properties of the invariance relationship between relations in a given domain and transformations acting as substitution rules in that domain. I planned this study bearing in mind that the terms - relations and transformations involved in invariance relationships are not isolated items; rather, they are elements of well-defined algebraic structures - fields of mq-sets for relations and semigroups of q-permutations for transformations - which are equipped with definite criteria for comparing, transforming and combining elements in their domains. This led me to inquire into the hereditary rules of the invariance relationship, along the lines of the structures forming the one and the other sides of the invariance paradigm. Elementary concepts from abstract algebra were helpful in describing and exploring those aspects.

Proofs and examples in this paper have been constructed with prevailing reference to the finite case, i.e., relations of various degrees acting in a set of values or entities which is finite in its cardinality. This choice corresponds to one of the purposes I had in addressing myself to the present study, i.e., to examine the aspect of the invariance problem in a finite set-theoretic environment and to discuss some of the questions which this special application may involve; in particular, the need for replacing the standard concept of a permutation with the weaker notion of a quasi-permutation. It may be seen, however, that the central results of my analysis still hold true when reference is made to relations in an infinite (rather than a finite) domain; in particular, there is no change, at the abstract level, concerning the rules of invariance and respectfulness preservation, which have been derived in Subsection 4.2 and are at the basis of the duality theory outlined in Section 5.

Some important problems are left unresolved in this paper - in particular, the characterization of fields of md-sets in the range of operator $\theta$ and that of semigroups of q-permutations in the range of operator $\tau$. There are also limitations, some of which depend on the particular kind of objects chosen as the specific target of my study. Here, we have considered relations of different possible degrees which may be defined in one given domain, but various other sorts of mathematical constructs may also be the objects of invariance problems, such as operations, relations between different domains, sets of various formal complexity, properties of forms in geometry, decision rules in statistics, quantitative laws in empirical science, and so on. Objects of these other kinds may also be viewed as members of suitably organized formal structures, so I conjecture that, in some respects, the method used here for discussing invariant relations may serve as a guide for discussing other sorts of objects within 
the framework of invariance theory.

Another limitation of this study is due to the reduced set of rules accepted as legitimate modes of forming new relations based on existing ones, these rules being the standard Boolean operators of complementation, intersection and union (and other operators such as set subtraction, Boolean sum, etc., which may be derived from them by formal combination). We must consider, in this regard, that the algebra of md-sets may be made more powerful by allowing other ways of operating on such sets, besides standard Boolean operators. The method of our analysis may be applied to explore such richer algebraic structures and their implications for the invariance problem.

As an example, I mention a pair of operations called "universal projection" and "existential projection", which are specified for all $X \subseteq V[N]$ and $J \subseteq N$ by the following equations:

$$
\text { universal projection of } X \text { on } J=\{s \in V[J]: s+t \in X \text { for all } t \in V[N \backslash J]\}
$$

existential projection of $X$ on $J=\{s \in V[J]: s+t \in X$ for some $t \in V[N \backslash J]\}$.

It may be seen that these two operations on md-sets cannot be expressed in Boolean terms and are the set-theoretic counterparts of universal and existential quantifiers, as they are defined in formal logic (cf. [Mendelson, 1964|). An algebra of md-sets which, besides Boolean operations, also comprises such projection rules would be strictly related to a first-order predicate calculus, and the theory of invariance as applied to such an algebra would be relevant for the invariance and meaningfulness problems of well-formed formulas in such a logical calculus.

\section{REFERENCES}

ADAMS E.W., FAGOT R.F., ROBINSON R.E, "A theory of appropriate statistics", Psychometrika 30, 1965, p. 99-127.

AIGNER M., Combinatorial theory, Berlin, Springer-Verlag, 1979.

BARBUT M., MONJARDET B., Ordre et classification. Algèbre et combinatoire, Tomes I et II, Paris, Hachette, 1970.

BICKEL P.J., DOKSUM K.A., Mathematical statistics: Basic ideas and selected topics, San Francisco (CA), Holden-Day, 1977.

BIGGS N.L., WHITE A.T., Permutation groups and combinatorial structures, Cambridge (IL), Cambridge University Press, 1979.

BIRKHOFF G., Lattice theory, Providence (RI), American Mathematical Society, 1967.

CASSIRER E., "The concept of group and the theory of perception", Philosophy and Phenomenological Research 5, 1944, p. 1-36.

CHIANG C.Y., "Measurement structures and invariant statistics", British Journal of Mathematical and Statistical Psychology 50, 1997, p. 43-54. 
DIXON J.D., MORTIMER B., Permutation groups, New York, Springer-Verlag, 1996.

DZHAFAROV E.N., "Empirical meaningfulness, measurement-dependent constants, and dimensional analysis", R.D. Luce, M. D'Zmura, D.D. Hoffman, G. Iverson, K. Romney (eds), Geometric representations of perceptual phenomena. Papers in honor of Tarow Indow on his 70th birthday, Hillsdale (NJ), Erlbaum, 1995, p. 113134

FERGUSON T.S., Mathematical statistics. A decision theoretic approach, New York, Academic Press, 1967.

FISHBURN P.C., ROBERTS F.S., "Uniqueness in finite measurement", F.S. Roberts (ed.), Applications of combinatorics and graph theory to the biological and social sciences, New York, Springer-Verlag, 1989, p. 103-137.

GUÉNOCHE A., "Construction du treillis de Galois d'une relation binaire", Mathématiques, Informatique et Sciences humaines 109, 1990, p. 41-53.

HALMOS P., GIVANT S., Logic as algebra, Washington (DC), Mathematical Association of America, 1998.

HOWIE J.M., An introduction to semigroup theory, London, Academic Press, 1976.

IVERSON G., LUCE R.D., "The representational measurement approach to psychophysical and judgmental problems", M.H. Birnbaum (ed.), Measurement, judgment, and decision making, San Diego (CA), Academic Press, 1998, p. 1-79.

KAYE R., MACPHERSON D. (eds), Automorphisms of first order structures, Oxford (UK), Oxford University Press, 1994.

LEHMANN E.L., Testing statistical hypotheses, New York, Wiley, 1959.

LUCE R.D., "Dimensionally invariant numerical laws correspond to meaningful qualitative relations", Philosophy of Science 45, 1978, p. 1-16.

LUCE R.D., KRANTZ D.H., SUPPES P., TVERSKY A., Foundations of measurement, Representation, axiomatization, and invariance, Vol. 3, New York, Academic Press, 1990.

MANDERS K.L., "On JND representations of semiorders", Journal of Mathematical Psychology 24, 1981, p. 224-248.

MENDELSON E., Introduction to mathematical logic, Princeton (NJ), Van Nostrand, 1964.

MUNDY J.L., ZISSERMAN A. (eds), Geometric invariance in computer vision, Cambridge (MA), MIT Press, 1992.

MUNDY J.L., ZISSERMAN A. (eds), Applications of invariance in computer vision, New York, Springer-Verlag, 1994.

NARENS L., "A general theory of ratio scalability with remarks about the measurement-theoretic concept of meaningfulness", Theory and Decision 13, 1981, p. 1-70.

NARENS L., Abstract measurement theory, Cambridge (MA), MIT Press 1985. 
NARENS L., "Meaningfulness and the Erlanger program of Felix Klein", Mathématiques Informatique et Sciences humaines 101, 1988, p. 61-71.

NARENS L., Theories of meaningfulness, Mahwah (NJ), Erlbaum, 2002.

PFANZAGL J., Theory of measurement, Würzburg, Physica-Verlag, 1968.

ROBERTS F.S., Measurement theory, Reading (MA), Addison-Wesley, 1979.

PIZLO Z., "A theory of shape constancy based on perspective invariants", Vision Research 34, 1994, p. 1637-1658.

SIKORSKI R., Boolean algebras, Berlin, Springer-Verlag, 1964.

VAN GOOL L.J., MOONS T., PAUWELS E., WAGEMANS J., "Invariance from the Euclidean geometer's perspective", Perception 23, 1994, p. 547-561.

WALSH V., KULIKOWSKI J. (eds), Perceptual constancy: Why things look as they do, Cambridge (UK), Cambridge University Press, 1998.

WEITZENHOFFER A., "Mathematical structures and psychological measurement", Psychometrika 16, 1951, p. 387-406.

WILLE R., "Restructuring lattice theory: An approach based on hierarchies of concepts", I. Rival (ed.), Ordered sets, Dordrecht, Reidel, 1982, p. 445-470.

YOUNES L., Invariance, déformations et reconnaissance de formes, Berlin, SpringerVerlag, 2004. 